\title{
Der Formenkreis Caementella loricata Borgert und seine Beziehungen zu Protocystis.
}

\author{
Aus dem Nachlaß von Prof. W. Mielck, \\ zusammengestellt von \\ AnNeliese Stier.
}

(Aus der Biologischen Anstalt auf Helgoland.)

(Mit 17 Abbildungen im Text.)

\section{Inhaltsverzeichnis.}

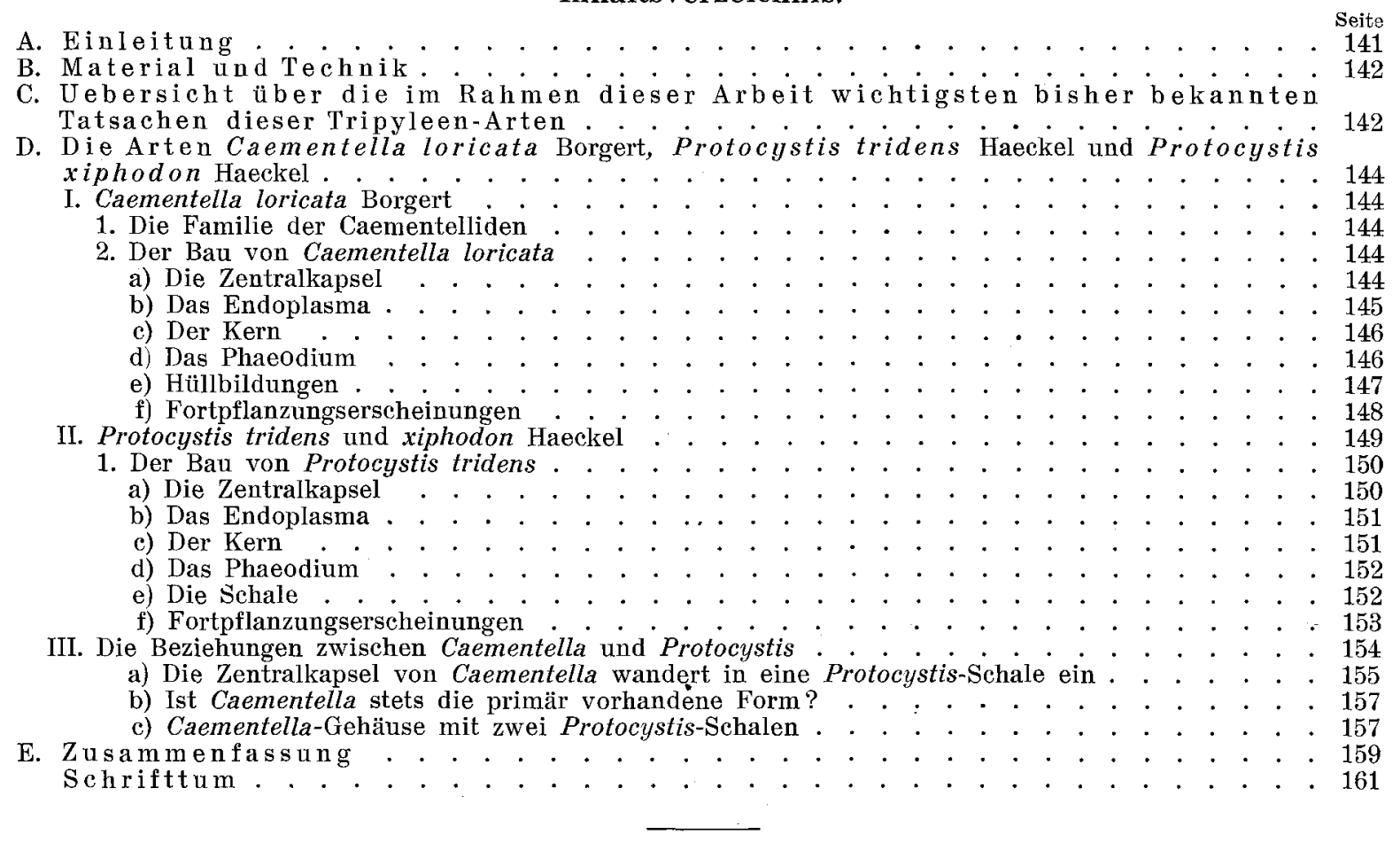

\section{A. Einleitung.}

Die vorliegende Arbeit war von Prof. Mrelck als eine weitere Spezialuntersuchung an Nordseeprotisten geplant, von denen 1912 als erste die Monographie von Phaeocolla pygmaea erschienen ist. Die Untersuchung beschäftigt sich mit Caementella loricata Borgert, einer Tripyleenart, die wohl noch eines eigenen Skelettes entbehrt, sich aber ein Gehäuse aus fremden Skeletten oder Skeletteilen aufbaut, sowie mit Protocystis und den Beziehungen dieser beiden Arten untereinander. Die Arbeit war als teilweise druckfertiges Manuskript vorhanden, als Prof. Mielck im Herbst 1933 starb. Die Bearbeiterin dieser nachgelassenen Untersuchungen arbeitete damals gerade für ihn an seinem reichen Radiolarienmaterial und fertigte unter anderem auch Zeichnungen für die vorliegende Arbeit an. Herr Prof. HAgmeier vertraute mir liebenswürdigerweise das Manuskript zur Fertigstellung an, wofür ich ihm an dieser Stelle nochmals danke. Die Teile der Untersuchungen, 
die schon in vollständiger Bearbeitung vorlagen, sind unverändert geblieben - der MIELCK'sche Originaltext ist stets in Anführungszeichen gesetzt worden - während andere Abschnitte nur aus vorhandenen Notizen zusammengestellt werden konnten (Einleitung, das Kapitel über die Beziehungen von Caementella zu Protocystis). Außer dem Manuskript waren ein Teil der Präparate und zahlreiche Skizzen für die vorliegende Arbeit noch vorhanden und wurden als Unterlagen für die Zeichnungen benutzt. Leider mußte auf eine nähere Untersuchung von Kern und Plasma, sowie der "Chromidienbläschen" bei Caementella loricata aus Mangel an frischem Material verzichtet werden. Aus demselben Grunde konnten die Untersuchungen an Protocystis xiphodon - Präparate hierzu waren kaum, Aufzeichnungen überhaupt nicht vorhanden - nicht ergänzt werden. Dagegen fand sich von Protocystis tridens noch reichlich Material, das von mir besonders für Kernfärbungen verwendet wurde.

\section{B. Material und Technik.}

Das Material stammt zum größten Teil von den Terminfahrten in der Nordsee, die in den Jahren 1906 und 1907 gemacht worden sind, und zwar fast ausschließlich von den Stationen N 7-N 10, d. h. also zwischen $63^{\circ}-64^{3}$ Nord und zwischen $2^{\circ}-5^{0}$ West. Eine Karte mit genauer Angabe der Terminstationen gibt MielCK in seiner Arbeit über "Heliozoa und Radiolaria“ 1913. Die Fänge wurden im August, Januar, Februar und März mit einem Vertikalnetz in $290-0 \mathrm{~m}$ Tiefe gemacht. Dazu kommt noch ein Fang, der im März 1930 in der Nähe der Vikingbank gemacht wurde, es handelt sich ebenfalls um einen Vertikalfang von 91,5- $0 \mathrm{~m}$ Tiefe. Ergänzend hierzu wurde von mir noch ein Fang ausgewertet, der im Oktober 1935 etwa $65^{\circ}$ Nord, $3^{\cup}$ West gemacht wurde, das entspricht bei den Terminfahrten den Stationen N 35-36. Allerdings fand sich hier nicht das erhoffte Caementella-Material, aber in großen Mengen Protocystis tridens. Der Fang wurde mit dem Apstein-Netz in einer Tiefe von $300-0 \mathrm{~m}$ gemacht. Es handelt sich also bei allen Fängen um Vertikalfänge, es läßt sich deshalb über die vertikale Verbreitung der Arten in diesen Gebieten nur soviel aussagen, daß ihre obere Verbreitungsgrenze noch höher als $91 \mathrm{~m}$ liegen muß. Da alles vorhandene Material nicht für spezielle cytologische Untersuchungen gesammelt worden war, ist es nur in Alkohol konserviert, ohne vorherige besondere Fixierung. Es sind deshalb alle cytologischen Beobachtungen an diesem Material nur von begrenztem Werte. Da aber ja bei allen vorhandenen Tieren die gleichen Veränderungen im Bau von Plasma und Kern vorliegen müssen, ist wohl eine ausführliche Beschreibung auch der cytologischen Befunde gerechtfertigt. Die von Prof. MIELcK angefertigten Präparate waren mit Haematoxylin EHRLicH und Eosin oder mit Paracarmin gefärbt. Das von mir noch nachträglich hinzugenommene Material (März 1935) wurde zum großen Teil ebenfalls mit Haematoxylin EHRLich und Eosin gefärbt, zum Teil auch mit Haematoxylin n. Weigert. Für die spezielle Untersuchung der Kernverhältnisse von Protocystis tridens wurde mit sehr guten Resultaten Feulgens Nuklealfärbung angewandt. Die Färbung wurde genau nach Vorschrift gemacht, nur wurde die Einwirkungsdauer der fuchsinschwefligen Säure auf zwei Stunden ausgedehnt. Es wurden auf diesem Wege von mir auch Untersuchungen über die Herkunft der für diese Tripyleenfamilie eigentümlichen "Chromidienbläschen" an Phaeocolla pygmaea gemacht. MIELCK hat die fraglichen Gebilde gerade an dieser Art eingehend untersucht und beschrieben (1912). - Da es sich bei dem vorhandenen Beobachtungsmaterial über die Beziehungen von Caementella und Protocystis nur um wenige Einzelexemplare handelt, ist es auch jetzt noch nicht möglich, die Beziehungen dieser beiden Arten zueinander lückenlos zu klären. Es muß deshalb des öfteren vorläufig bei Vermutungen bleiben. Immerhin sind die Stadien, die auf einen engen Zusammenhang dieser beiden Tripyleenarten hinweisen, so häufig, daß derselbe nunmehr als Tatsache gelten muß.

\section{c. Uebersicht über die im Rahmen dieser Arbeit wichtigsten bisher bekannten Tatsachen dieser Tripyleen-Arten.}

Eine von MieLck geplante kurze Uebersicht über die bisher bekannten wichtigsten Tatsachen des Baues und der Entwicklung dieser Tripyleenfamilie soll zu seinen eigenen Beobachtungen überleiten. Es wird an dieser Stelle jedoch nur das herausgegriffen werden, was von besonderer Wichtigkeit für die systematische Stellung dieser Familie ist, während alle anderen an diesen Tieren bereits gemachten Beobachtungen erst vergleichend mit den 
vorliegenden gebracht werden sollen. EHRENBERG und auch noch HAECkEL (1862) sahen in den Skeletten von Silicoflagellaten Radiolarienskelette. R. HerTwiG fand als erster Caementelliden (1879), deren Gehäuse aus Skeletten von Dictyocha bestanden, und nahm deshalb an, daß es sich bei den kleinen Skelettelementen nicht um selbständige Radiolarienskelette handle, sondern um Teile eines Tripyleengehäuses, nämlich um das der ihm vorliegenden Caementelliden. Er nannte diese Tripyleenart deshalb Dictyocha und schloß sie an die Familie der Aulacanthiden an. HaEckel teilt (1887) HERTwigs Auffassung und spricht die von ihm gefundenen Arten als zur Familie der Cannorraphiden gehörig an. Nachdem Borgert (1891) nachgewiesen hatte, daß Dictyocha und Distephanus im Sinne Ehrenbergs doch selbständige Tiere sind, und zwar sogen. Silicoflagellaten, erkannte er in den Caementelliden Tripyleen mit einem Fremdkörperskelett, die die genannten Silicoflagellaten zum Aufbau desselben benutzen. Er teilte die erst von ihm als Caementelliden bezeichnete Familie (1909) auf, und schlieft sie den Phaeodiniden an, denjenigen Tripyleen also, die keinerlei Skelettelemente aufweisen und deshalb allgemein als die primitivsten Tripyleen überhaupt gelten. Ein Vertreter dieser einfachsten Radiolarien wurde von MIELCK (1912) einer genauen Untersuchung unterzogen, es handelt sich um die bereits oben erwähnte Monographie über Phaeocolla pygmaea. Mit Caementella macht BORGERT in einer Arbeit über die Ergebnisse der Plankton-Expedition (1889) näher bekannt (1909). Als wichtigstes Resultat von einschneidender Bedeutung war der Fund von multipler Teilung. Erst hierdurch wurde sichergestellt, daß es sich bei diesen primitiven Tripyleen wenigstens teilweise um selbständige Arten handeln muß, zu denen also auch Caementella gehört. BORGERT selbst hatte nämlich vorher $(1891,1901)$ angenommen, daß es sich bei den Phaeodiniden und Caementelliden nur um Jugendstadien skelettführender Arten handelt, die sich wohl durch Zweiteilung - die schon lange bekannt war - vermehren können, denen aber andere Fortpflanzungsmöglichkeiten fehlen. BORGERT nimmt in Anbetracht der ganz ähnlichen Verhältnisse bei Aulacantha an, daß es sich bei dem von ihm gefundenen Fall von multipler Teilung um Gametenbildung handelt. Die Familie der Caementelliden enthält deshalb mutmaßlich verschieden entwicklungsfähige Formen, von denen die einen stets ein Fremdkörperskelett behalten (Formen mit Gametenbildung), während andere nur Jugendstadien von Formen mit eigenem Skelett sind. - $\mathrm{Zu}$ erwähnen sind noch HAECKERs und SchröDERs Auffassungen über diese Familie. HAECKER vertrat vor BorgerTs Fund der Gametenbildung bei Caementella den Standpunkt, daß es sich bei den nahe verwandten Phaeodiniden (und zwar bei den dicystinen Formen) um Jugendstadien von dicystinen Tripyleen mit Eigenskelett handeln müsse, und zwar um solche von Aulacanthiden. $\mathrm{Da}$ er die Caementelliden ebenfalls für derartige Jugendstadien hält, beginnt seine Tripyleensystematik erst mit der Familie der Aulacanthiden. - O. SchröDer gibt als erster Abbildungen, die tatsächlich auf eine Beziehung von Caementella und Protocystis hinweisen, es handelt sich dabei seiner Ansicht nach um Protocystis tridens. Zentralkapsel und Kern sind, seinen Beobachtungen nach, so ähnlich bei diesen beiden Arten, daß sie ohne das sie umgebende Skelett nicht $\mathrm{zu}$ unterscheiden wären. Allerdings sei dỉe Struktur des Kernes bei Protocystis tridens eine andere als bei Caementella loricata. Er fand jedoch Exemplare (vergl. seine Abb. 5, T. XXXII, 1913) von Protocystis tridens, die am Peristom Reste eines Caementellidengehäuses trugen, andererseits Exemplare von Caementella, die eine große gefaltete Membran enthielten, die als Anlage eines Protocystisgehäuses gedeutet werden kann. Er hält deshalb einen inneren Zusammenhang dieser Erscheinungen für sehr wahrscheinlich. SchröDER schließt aus seinen Funden, daß die Caementelliden Teilungsstadien von kleinen Protocystisarten sind, oder aber den Challengeriden sehr nahe verwandte Formen, die letztgenannte, einschränkende Deutung, die ja die Funde nicht erklärt, wird nicht begründet.

Es sind also mehrere schwer zu vereinende Ergebnisse der vorhandenen Untersuchungen da, die einer näheren Erklärung harren. Einmal tritt neben der Zweiteilung bei Caementella multiple Teilung (Gametenbildung nach BorGERT) auf, die darauf hinweist, daß wir es hier mit einer selbständigen Familie zu tun haben. Zum anderen sind Stadien gefunden worden, die Protocystis und Caementella in einem so engen Zusammenhang zeigen, daß an eine Zufälligkeit dieser Erscheinung nicht gedacht werden kann. Dabei ist es aber z. T. nicht zu unterscheiden, welches der beiden Individuen das primär vorhandene ist. Es gibt zwei Erklärungsmöglichkeiten: Entweder besteht ein Generationswechsel zwischen Caementella und Protocystis, und die Zentralkapsel der ersteren tritt in die neugebildete Schale der kleinen Challengeride über, oder aber die Caementella entsteht aus einem Teilungsstadium von Protocystis, sodaß die Tochterindividuen von Protocystis Caementelliden sind. 
Die folgenden Untersuchungen MIELCKs sollen zur Klärung dieser Frage beitragen können diese aber auch noch nicht vollständig lösen.

\section{Die Arten Caementella loricata Borgert, Protocystis tridens Haeckel und Protocystis xiphodon Haeckel.}

\section{Caementella loricata Borgert.}

„Ueber den Bau dieser in unserem Meeresgebiete zum ersten Male beobachteten, aber keineswegs seltenen Formen kann ich bezüglich der Zentralkapsel weniger eingehend berichten als über Phaeocolla pygmaea, da sich die Zentralkapsel infolge der Art der Gehäusebildung und durch das sie umgebende Phaeodium der Beobachtung oft entzieht und Schnitte nicht angefertigt werden konnten. Es kam mir bei dieser Untersuchung auch vor allem auf die Feststellung der Beziehungen Caementella-artiger Formen zu Protocystis an."

\section{Die Familie der Caementelliden.}

„Die Familie der Caementelliden wurde, wie bereits oben erwähnt, von BoRGERT (1909 b) aufgestellt, wobei der Bau dieser interessanten kleinen Tripyleen bereits von dem Autor recht ausführlich beschrieben wird. Ich beschränke mich deshalb auf die Beschreibung der Formen, wie sie mir vorlagen, und verweise im übrigen auf Borgert (1909 b). BORGERT rechnet mit der Möglichkeit, daß seine Caementellidenfamilie sowohl besondere selbstständige Arten umfaßit, die stets nur eine Fremdkörperhülle besitzen, als auch jugendlich e Stadien andere Arten, die später ein eigenes Skelett ausscheiden (1909b, 1922). Daß BORGERT in seiner Annahme, jedenfalls was den zweiten Punkt betrifft, z. T. Recht hat, soll in einem späteren Abschnitt der vorliegenden Arbeit gezeigt werden.

BORGERT faßt fünf verschiedene Formen unter dem Namen Caementella loricata zusammen, da einerseits eine Unterscheidung einzelner Species auf Grund der Verschiedenheit der aus dem mannifaltigsten Fremdkörpermaterial gebauten Hüllbildungen nicht statthaft ist, andererseits aber die Abgrenzung einzelner Arten auf Grund hinreichender Unterschiede noch nicht gelang. Von diesen fünf BoRGERT'schen Formen stimmt mit den mir vorliegenden am besten die Nr. 5 (T. XXI, Fig. 3) überein; dieser Figur genau entsprechende Individuen habe ich oft gesehen. Ferner traten ähnliche Formen auf wie Nr. 1 (T. XXII, Fig. 4), nur daß Dictyocha nicht so vorherrschend war. So große Exemplare wie Nr. 3 und Nr. 4 habe ich nie gesehen. Die von mir untersuchten Caementelliden zeigten alle große Uebereinstimmung in Bau und Größe, so daß sie zur Zerlegung in mehrere Arten keine Veranlassung boten. Die Zusammensetzung des Fremdkörperskelettes kann nach BoRGERTs Auffassung, wie eben erwähnt, zur Unterscheidung verschiedener Arten nicht herangezogen werden. Sie ist lediglich von dem Artenreichtum der Umgebung abhängig. Dafür spricht vor allem, daß das Gehäuse z. T. aus ganz verschiedenartigen Teilstücken zusammengebaut ist, andererseits gibt es auch Tiere, deren Panzer fast ausschließlich aus Dictyocha, Distephanus, Nassellarien oder CoscinodiscusArten besteht, wenn eben diese Arten im umgebenden Plankton vorherrschend waren."

\section{Der Bau von Caementella loricata Borgert.}

a) Die Zentralkapsel.

"Die Zentralkapsel zeigt meist kugelige bis ellipsoide Gestalt. Wie bei Phaeocolla pygmaea hat sich das Endoplasma infolge der Konservierung gewöhnlich etwas von der feinen Zentral ka p selmembran zurückgezogen, die daher an den präparierten Stücken eine abstehende, faltige Hülle bildet. Die doppelte Natur derselben konnte ich nicht mit Sicherheit feststellen. Bei freigelegten Zentralkapseln gelang es nur in den seltensten Fällen eine Astropyle festzustellen, die sich an einem Pole des kurzen Durchmessers der ellipsoiden Zentralkapsel befand. Ueber ihren Bau vermag ich genaueres nicht anzugeben. Sie erschien als eine einfache, kurz trichterförmig vorgezogene Oeffinung der Membran, die am Grunde oder im ganzen Verlauf eine feine Längsstreifung erkennen läßt. Ein dentlich abgesetztes „Operkulum“ wurde nicht erkannt. Zwar zeigt sich in der Umgebung der Oeffnung am Fuße der kurzen Proboscisbildung der Membran eine radiäre Streifung, doch kommt diese wohl durch Faltung der Membran selbst zustande, auch konnte von einer Verdickung der Membran an dieser Stelle nichts bemerkt werden. 
Parapylen gelangten überhaupt nicht zur Beobachtung. Die BoRgERT'schen Caementelliden (1909 b) besaßen stets eine Astropyle; Parapylen konnte BORGERT nur bei einer besonders großen Form mit voller Sicherheit nachweisen."

\section{b) Das Endoplasma.}

Das Endoplasma besitzt hier niemals ein Endophaeodium, das bei Phaeocolla pygmaea stets vorhanden war; es zeigt einen alveolenreichen Bau mit zahlreichen größeren und regelmäßigeren Alveolen als bei der eben genannten Phaeodinide. - Eine besondere, im Endoplasma des öfteren auftretende Erscheinung verdient noch erwähnt zu werden. Es finden sich nämlich in einem Teil der Alveolen verschiedentlich Einschlüsse, die Aehnlichkeit mit dem von Mielck (1912) für Phaeocolla pygmaea beschriebenen Inhalt der "Chromidienbläschen" haben, vergl. Abb. 21. Sie sind ebenfalls mit Kernfarbstoffen lebhaft gefärbt. Allerdings handelt es sich hier nur um wenige runde Granula, während die von MiвLCK gegebenen Bilder mehr an die Struktur akzessorischer Kerne erinnern, vergl. MiELCK 1912 Tafel XIV Fig. 7-12. Man wird bei diesen Gebilden auch an die von BORGERT beschriebenen, bläschenförmigen Einschlüsse erinnert, die bei den vegetativen Teilungsprozessen von Aulacantha auftreten, die aber im Gegensatz zu den vorliegenden Gebilden stets entweder ungefärbt bleiben oder aber nur Eosin annehmen. Sie müssen also grundsätzlich anderer Natur sein. Mit den von BORGERT beschriebenen abortiven Chromidien haben die Gebilde im Endoplasma von Caementella keinerlei Aehnlichkeit. Es läßt sich auch ein Zusammenhang der "Chromidienbläschen" mit Fortpflanzungsvorgängen nicht feststellen, da die fraglichen Gebilde sich einerseits in Individuen befanden, deren Kerne im Ruhestadium waren, andersseits aber auch in Teilungsstadien. Es fanden sich aber desgleichen Fortpflanzungsstadien, denen sie vollständig fehlten (vergl. Abb. 3). Auffällig häufig finden sie sich bei jungen Zentralkapseln, die man an der geringen Größe und den wenigen großen Alveolen gut als solche erkennen kann (s. Abb. 1). Als besonders charakteristisch schreibt MIELCK für Phaeocolla pygmaea, daß die Gebilde stets nur im Endoplasma anzutreffen sind. Sie liegen im oralen Teil der Zentralkapsel im Endophaeodium, und zwar immer in der Nähe des Kernes. Sie sind jedoch im oralen Teil der Zentralkapsel nie dort anzutreffen, wo die Astropyle liegt, sondern sind von derselben immer durch Phaeodellen getrennt. Es ist deshalb nicht möglich, daß sie von außen stammen. Im aboralen Teil der Zentralkapsel sind sie nie anzutreffen. Vergleicht man die für Phaeocolla pygmaea charakteristischen Merkmale mit denen von Caementella loricata, so erkennt man, daß letztere ebenfalls nur im Endoplasma vorkommen und sich mit Kernfarbstoffen färben lassen. Für Phaeocolla konnte mit Hilfe von Feulgens Nuklealreaktion festgestellt werden, daß es sich bei den "Chromidienbläschen" tatsächlich um Kernderivate handelt, während die Untersuchungen "an Caementella aus Mangel an frischem Material leider unterbleiben mußten. Wenn die Phaeodellen nun tatsächlich mit den "Chromidienbläschen" in einem ursächlichen Zusammenhang stehen, wie es Mielck für Phaeocolla auf Grund seiner Befunde annimmt, so müßte man meinen, daß die letzteren sich bei Caementella besonders häufig in der Nähe der Astropyle befinden müßten, da Caementella ja nur ein Ektophaeodium besitzt. Diese Vermutung hat sich aber nicht bewahrheitet, die "Chromidienbläschen" sind in einer Anzahl der Alveolen verteilt, ohne daß sich irgendwelche besonderen Lagebeziehungen zur Astropyle oder zum Kern feststellen lassen. Es ist aber immerhin möglich, daß sich derartige Stadien noch finden werden. Es wäre dann damit der Nachweis erbracht, daß die Phaeodellen tatsächlich ein Gebilde des Endoplasmas sind, und - wie mit FEULGeN nachweisbar - sogar in Kernderivaten ihren Ursprung haben. Eine weitere Untersuchung der Verhältnisse wäre auch besonders an gut fixiertem Material sehr wünschenswert, da es sich auf diese Weise ermöglichen ließe, einige noch ungelöste Fragen über Herkunft und Bedeutung der Phaeodellen zu klären. - 


\section{c) Der Kern.}

„Der Kern hat die gleiche Gestalt wie die Zentralkapsel und besitzt zum Unterschied von Phaeocolla pymaea zentrale Lage und auch bedeutendere Größe. Hinsichtlich der Struktur wurden verschiedene Bilder beobachtet. Am häufigsten gelangte eine schon von Borgert (1909b) T. XXII, Fig. 5 abgebildete ziemlich grobschollige, gleichmäßige Verteilung des Chromatins zur Beobachtung, nicht selten aber auch die bei Aulacantha und anderen Tripyleen vorkommende und von HAECkER bei Schnitten als "Radstruktur" bezeichnete Anordnung, wo das Chromatin radiär nach allen Seiten in dicken verästelten Strängen oder in kurz zylindrischen Stücken ausstrahlt (vergl. auch BorgeRT 1909 b, T. XXII, Fig. 6), s. Abb. 2, 5. Einige Male gewährte der Kern ein ganz ähnliches Bild wie bei Phaeocolla pygmaea: eine größere Ansammlung des Chromatins lag einer etwas abgeplatteten Seite des Kernbläschens an; von hier aus strahlte es radiär in Brocken oder Strängen auf die gewölbtere Seite aus. In dem hier vorliegendem Falle haben wir es wohl mit einem vorübergehendem Zustande zu tun, indem nach der Teilung der Kern sich noch nicht abgerundet und auch noch kein neues Chromatinzentrum gebildet hat, vergl. Abb. 3, 5. Auf weitere Kernverhältnisse werde ich in einem späteren Abschnitt über Fortpflanzungsverhältnisse etwas näher eingehen. Das Vorhandensein einer Kernmembran konnte nicht immer festgestellt werden."

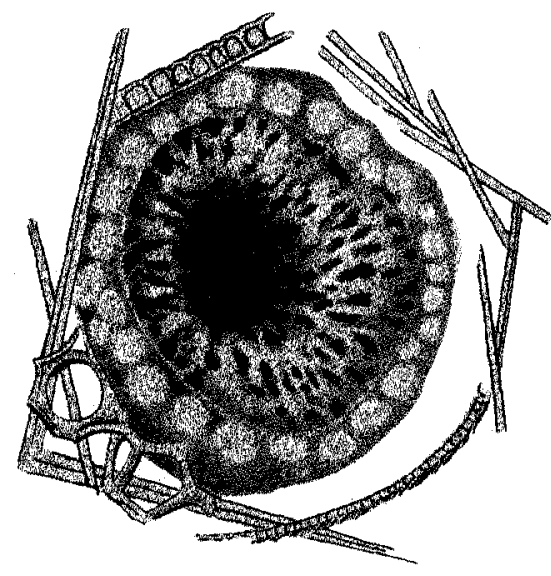

Abb. 2. Caementella loricata: „Radstruktur“ des Kernes. Haem. Ehrlich. Vergr. 840 メ.

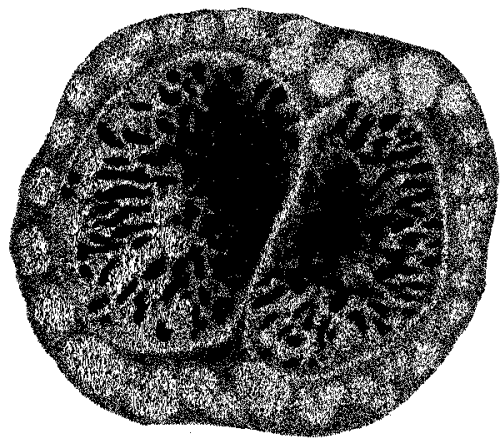

Abb. 3. Caementella loricata „Direkte Kernvermehrung" (n. BorGERT). Haem. EHRLICH, Eosin. Vergr. $840 \times$.

\section{d) Das Phaeodium.}

-Bei Caementella findet sich niemals ein Teil des Phaeodiums innerhalb der Zentralkapsel wie bei Phaeocolla pygmaea. Die Phaeodellen bilden eine einheitliche Masse außerhalb der Zentralkapsel an der durch die Astropyle bezeichneten oralen Seite. Das Phaeodium besitzt im Gegensatz zum Ektophaeodium von der eben genannten Phaeodinide einen bestimmten, beschränkten Platz und nimmt einen eng umschriebenen Raum für sich in Anspruch. Während bei Phaeocolla pygmaea zwischen den Ektophaeodellen eine Anzahl wohl zum Schutze aufgenommener, größerer, kieseliger Körper (Röhren und Scheiben von Diatomeengehäusen, Chaetoceras-Hörner etc.) und anderes gröberes Material (Tintinnen-Gehäuse etc.) ihren Platz finden, treffen wir im Phaeodium von Caementella loricata keine größeren kieseligen Beimengungen an, sondern nur allerkleinste Diatomeen und andere Teilchen, die vermutlich als Nahrung aufgenommen worden sind. Es ist überhaupt wohl kaum statthaft, das Phaeodium von Caementella nur mit den Ektophaeodellen von Phaeocolla pygmaea in Uebereinstimmung zu bringen, weil möglicherweise letztere bereits ihre dem Stoffwechsel dienenden Zwecke erfüllt haben und nur als Bausteine für das Kalymma weiter verwandt werden. Das Phaeodium ist regelmäßig durch eine freie, helle Schicht, die vielleicht nur dem Zurückschrumpfen der Zentralkapsel infolge der Konservierung ihre Entstehung verdankt, von der letzteren getrennt. Der Umfang des Phaeodiums ist wechselnd, gewöhnlich hällt es die Zentralkapsel an einer Seite wie eine Wolke oder eine Kappe ein. Die Phaeodellen selbst weichen nicht von den sonst bei den Tripyleen geschilderten ab. 
Sie sind auch hier verschieden grof, faltig und infolge höheren oder geringeren Gehaltes an Pigmentkörnern bald dunkler, bald heller. Man findet schwach färbbare von bräunlichem Grundton meist geringerer Größe und größere, die in höherem Maße Farbstoff annehmen. Ebenso kommen die bekannten, auch bei Phaeocolla pygmaea festgestellten gefalteten Membranen vor, kleinere im Phaeodium selbst und größere, die daraus hervorragen oder auch frei in der Gehäusehülle liegen. Zuweilen beobachtet man sackförmige Membranen, die aus der Hülle frei hervorhängen und reichlich Eosin annehmen. Vielleicht hat man in solchen Bildungen die häutige, noch gänzlich der Struktur entbehrende Grundlage des zu bildenden Protocystis -Gehäuses vor sich (s. u.). Derartige Gebilde erwähnt schon SCHRöDER 1913. T. XXIII, Fig. 4."

\section{e) Hüllbildungen.}

„Im Bau der Hüllbildungen zeigen die mir vorliegenden Stücke große Uebereinstimmung mit der BoRGERT'schen Caementella. Die von außen aufgenommenen, kieseligen Fremdkörper bilden einen das Extrakapsularium überkleidenden Panzer, indem sie zur Oberfläche der Zentralkapsel und des Phaeodiums eine vorwiegend tangentiale Anordnung annehmen. Diese bald lockere, bald dichtere Hülle besitzt infolge der tangentialen Anordnung ihrer Bestandteile wie die Zentralkapsel eine im ganzen kugelige bis ellipsoide Grundform, die aber je nach dem zur Verwendung gekommenen Material von dieser Form etwas abweichen kann. Oft beobachtet man eine korbartige Gestalt der Hülle, wobei der "Korb" selbst von den verschiedenartigsten kieseligen Fremdkörpern, vornehmlich in Scheiben- und Tellerform gebildet wird. Die Oeffnung aber nimmt das Phaeodium ein, das seine Oberfläche wiederum mit einem Nadelfilz von Chaetocerashörnern oder anderen stäbchenförmigen Diatomeen bedeckt hat. Solche Formen sind vermutlich infolge der Teilung zustandegekommen: das ursprünglich geschlossene Gehäuse hat sich geteilt, so dafs die beiden Teilgehäuse zunächst an einer Seite offen stehen und daher eine korbartige Gestalt annehmen (s. Abb. 4). Da die Teilung in der Linie zwischen den einander zugewandten Phaeodien der beiden Zentralkapseln erfolgt (vergl. S. 13), so liegt das Phaeodium nach der Teilung an der offenen Seite des Gehäuses und schützt die Zentralkapsel einstweilen vor äußeren Einflüssen. Die Oberfläche erhält dann zunächst eine Decke aus kleinen nadelartigen Bestandteilen und erst später wird die Hülle durch Aufnahme von größeren scheibenförmigen oder hutartigen Körpern wieder völlig geschlossen. Die Hülle setzt sich vorzugsweise aus kleinen Coscinodiscus- bezw. Thalassiosira-Schalen und aus Silicoflagellaten-Gehäusen zusammen, entweder zu gleichen l'eilen oder indem bald diese, bald jene den Hauptanteil nehmen oder auch ausschlieBlich benutzt werden. Es kommt dabei darauf an, welches Baumaterial in größerer oder geringerer Menge im Plankton vorhanden ist. So benutzten die im A u gust 1907 Stat. DN 7 gefundenen Caementellen vorzugsweise Silicoflagellatengehäuse, die vom Februar 1907 Stat. DN. 7; DN 8; N 9 (vergl. die Karte Miesck 1913), mehr Coscinodiscus-Schalen. Von den letzteren wurde besonders Coscinodiscus radiatus verwendet. Coscinodiscus excentricus und

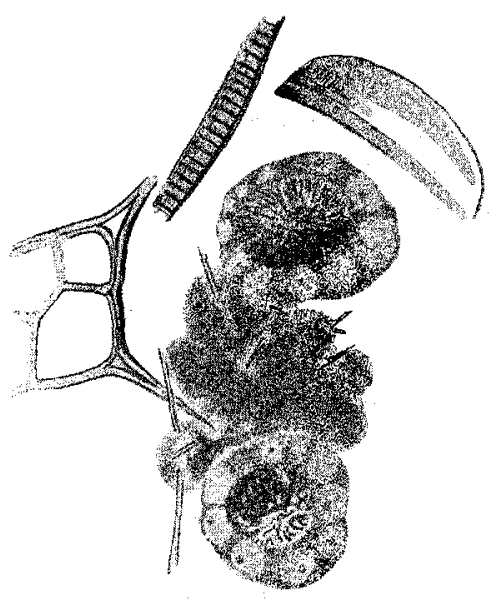

Abb, 4. Camentella loricata: Geteiltes Individium. Das Phaeodium liegtzwischen den beiden Zentralkapsein (vergl. Abb. 1). Haem. EHRLICH, Eosin. Vergr. $840 \times$ verschiedene, nicht näher bestimmbare Thalassiosiren, auch Actinoptychus und Pleurosigma, von Silicoflagellaten mehr Distephanus als Dictyocha und vereinzelt ein kleines Gehäuse (vergl. Abb. 5, 13), dessen Zugehörigkeit zu den Sillicoflagellaten ich vermute. ${ }^{\perp}$ Als Füllmaterial zwischen diesen scheiben- und hutförmigen Gebilden nehmen einen nicht unwesentlichen Anteil an der Hüllbildung sowohl tangential als auch radial gelagerte, lang röhrenförmige Schalenbildungen von Diatomeen wie Thalassiothrix, Bacillaria, Rhizosolenia und vor allem Endhörner und Zwischenhörner von verschiedenen Chaetoceras-Arten, dazu auch Borsten oder Schalenteile von Biddulphia. Gelegentlich kamen auch kurze Paralia-Ketten vor, die mit ihrer Pervalvarachse radiär

1) Es handelt sich um das von GemeinhardT 1931 beschriebene Skelett von Actiniscas, das dort T. IX, Abb. 9-11 abgebildet ist! 
gestellt waren. Auf das Vorkommen nach außen gerichteter Kieselstücke in den Gehäusen und die Bedeutung dieser Erscheinung für die Erhöhung der Schwebefähigkeit der Organismen hat BORGERT bereits hingewiesen (1909 b, p. 295 u. 1922, p. 44). Schließlich tragen noch die Radiolarien zur Lieferung der Baustoffe bei: kleine Spumellarien (Hexacontium, Rhizoplegma), Nasselarien (bes. Plagiacantha u. a. kleine korbförmige Arten), sowie Stachelbüschel und einzelne Stachel von Sticholonche. Das Material der Hüllbildung ist so mannigfaltig, so daß ich mich der BORGERT'schen Ansicht anschließe, daß wohl kaum von einer besonderen Auswahl die Rede sein kann. Je nachdem die eine oder die andere Form mehr im Plankton vorhanden ist, wird sie auch bei diesen Schalenbildungen vorzugsweise vertreten sein. Die Gröfe der Gehäuse ist sehr verschieden; es gibt Exemplare, die als Gehäuse nur die büchsenförmige Schale eines einzigen Coscinodicus bewohnen, so, daß die Büchse an der einen Seite geschlossen ist, während sie an der anderen Seite muschelartig klafft. Die Zentralkapsel ist dabei an der geschlossenen Seite gelegen, während die Oeffnung vom Phaeodium ausgefüllt wird, das an seiner Oberfläche mit Chaetoceras-Borsten bedeckt ist. Ein anderes Exemplar bewohnte das Skelett einer einzigen Spumellarie! Der weitaus größere Teil jedoch benutzt mehrere große Skeletteile, zwischen die dann kleinere eingestreut sind. Je nachdem diese Bauelemente aneinandergefügt werden, ergibt sich dann eine Gesamtform, die mehr rund oder kubisch ist, wie sie BorgerT abgebildet hat, s. $1909 \mathrm{~b}$, T. XXII Fig. 3, 4 und T. XXIII Fig. 4, 5.“

\section{f) Fortpflanzungserscheinungen.}

Das Material, welches Untersuchungen über Fortpflanzungserscheinungen zuließ, war nicht besonders reichlich, denn oft entziehen sich die Zentralkapsel und der Kern durch das Phaeodium und die Hüllbildungen - wie bereits oben erwähnt wurde einem tieferen Einblick. Zuweilen liefen sich jedoch die Zentralkapseln freilegen, oder es kamen welche zur Beobachtung, die ihre Hülle durch irgendeinen Zufall ganz oder zum Teil verloren hatten. An solchen Stücken ließen sich Zentralkapsel und Kern am besten beobachten. Es kam mir mehrmals folgendes Stadium vor die Augen: in einer Zentralkapsel befand sich ein langgestreckter Kern, in welchem die Chromatinmassen sich bereits in zwei Teile geteilt hatten, die nur durch einen ganz schmalen Streifen der chromatinlosen Substanz voneinander getrennt sind. Jede Chromatinhälfte hat bereits wieder begonnen, ein eigenes Chromatinzentrum zu bilden, von dem das Chromatin in einer Struktur ausstrahlt, die etwa mit der von HAECKER als "Radstruktur" bezeichneten (dort allerdings für Schnitte gebraucht!) übereinstimmt. Die Kernmembran zeigt in der Teilungsebene des Chromatins erst an der - nach der Lage des Phaeodiums zu schließen - aboralen Seite eine Einschnürung der Membran, während die andere Seite noch glatt ist. In einigen weiteren Stadien ist der Kern bereits völlig geteilt, aber die beiden Teilstücke zeigen an der der Teilungsebene zugewandten Seite noch eine starke Abplattung, sie sind auch nur durch einen schmalen Plasmastreifen voneinander getrennt, Abb. 3. Sie zeigten auf diesem Stadium entweder "Radstruktur" oder mehr homogen grobschollige Beschaffenheit. Die Zentralkapsel selbst zeigte auch hier noch keine Teilung. Weiterhin kam ein Stadium vor, wo von den schon mehr abgerundeten Teilstücken des Kernes der eine „Radstruktur“, der andere "Brockenstruktur" besitzt mit dichterer Ansammlung des Chromatins an der dem anderen Kern zugewendeten Hälfte. Diese Struktur wurde übrigens auch in einzelnen Kernen einfacher Zentralkapseln beobachtet; der Kern war dabei an einer Seite abgeplattet, und das Chromatin hatte sich an dieser platten Seite angesammelt und strahlte von hier auf den gewölbten Teil aus, so daß es das Aussehen hatte, als habe er nach eben erfolgter Teilung kein neues Chromatinzenzentrum gebildet, sondern die exzentrische Lage des Chromatins beibehalten. Wenn überhaupt eine Astropyle zu erkennen war, so konnte festgestellt werden, daß die Chromatinansammlung an der oralen Seite des Kernes lag, wie das bei Phaeocolla der normale Zustand ist, vergl. Abb. 5. Den Teilungsvorgang der Zentralkapseln nach der Kernteilung konnte ich nicht beobachten. Doch darf man aus der Form der mehrmals gesehenen Zentralkapseln, welche die Teilung eben beendet hatten und noch dicht mit ihren abgeplatteten Seiten aneinanderlagen, nur eine schmale Schicht Endoplasma zwischen sich lassend, auch auf Teilung durch Spaltung schließen. Die Kerne waren in diesen Stadien oft noch nicht ganz abgerundet und, besaßen entweder beide "Radstruktur", oder sie stimmten im Aussehen nicht überein, indem der eine mehr brockenartige, der andere mehr strangartige Struktur besaß. Während der Teilungsvorgänge konnte ich eine Astropyle nie erkennen. Das Phaeodium nahm während der Teilung den Platz vor der oralen Gegend der ursprünglichen Zentralkapsel ein. Die Vor- 
gänge gleichen sehr denen von BoRGERT an Aulacantha beobachteten, die er als „Zweiteilung mit direkter Kernvermehrung " bezeichnet.

„Die Zentralkapseln rücken dann auseinander, und Phaeodiummassen treten gleich zwischen sie, so daf man annehmen muß, daß die Astropylen einander entgegengerichtet werden. Zwei Zentralkapseln, in einer Hülle an den entgegengesetzten Seiten eines gemeinsamen Phaeodiums liegend, wurden häufig beobachtet, s. Abb. 4. Dann teilt sich auch dieses Phaeodium und sehließlich auch die Hülle in derselben Ebene wie die vorausgegangene Teilung des Kernes und der Zentralkapsel. Dabei entstehen zunächst die bereits oben erwähnten korbförmigen Hüllen, deren offene Seite vom Phaeodium eingenommen ist". Die in Abb. 4 dargestellten Kernstrukturen legen jedoch die Vermutung nahe, daß hier keine „direkte Kernvermehrung" im Sinne BORGERTS vorliegt, sondern eine Mitose vorausgegangen ist. Das zeigt besonders deutlich Abb. 1, das eine vergrößerte Zentralkapsel von Abb. 4 darstellt.

„Die Teilung kann anscheinend sehr lebhaft vor sich gehen, denn es wurde ein Stadium mit zwei Zentralkapseln beobachtet, in dem die eine bereits wieder zweikernig war. Auch kommt es nicht immer gleich zur sofortigen Teilung der Hülle, sondern die Befunde zeigen, daß die Teilprodukte zuweilen in einer gemeinsamen Hülle verbleiben. So fand sich ein Exemplar, welches fünf Zentralkapseln besaß, von denen ein Paar mit einander zugewandten, abgeplatteten Seiten sich gerade geteilt hat, während eine weitere Zentralkapsel sich eben teilt, s. Abb. 5 .

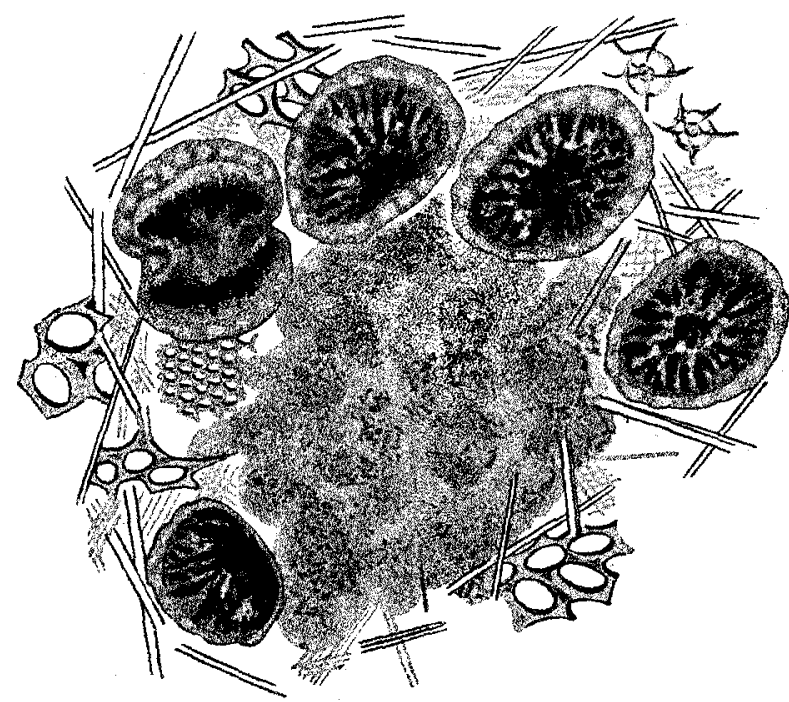

Abb. 5. Caementella loricata: Teilung eines Individuums in 6 Zentralkapseln. Es sieht aus, als ob sowohl direkte Kernvermehrung als auch mitotische 'Teilung vor sich gegangen sind. Paracarmin. Vergr. $420 \times$. Drei der Zentralkapseln ließen deutlich „Radstruktur" ihrer Kerne erkennen. Das Phaeodium liegt hier im Zentrum der Hülle, und die Zentralkapseln sind darum herum gruppiert. Eine derselben liegt etwas abseits und ist mit den umgebenden Hüllteilen etwas von den anderen losgelöst, sie scheint im Begriff $\mathrm{zu}$ sein, sich aus dem Verbande zu lösen.

Mehrfach wurden Exemplare in Stadien beobachtet, die von der oben und bereits von BORGERT für Aulacantha geschilderten Teilungsweise abweichen, und die das Vorkommen einer noch einfacheren Teilungsweise wahrscheinlich machen. In den Anfangsstadien waren Kern und Zentralkapsel sehr in die Länge gestreckt, dann machte sich in der Mitte der Zentralkapsel eine seichte Einschnürung bemerkbar, die immer tiefer vordrang und so unter Annahme von Hantel- oder Bisquitform schließlich zur Teilung führte. Solche Stadien wurden auch sonst zuweilen gesehen, jedoch kamen sie namentlich im Februar 1907 St. N 7 vor. All diese Stadien waren aber unter dem Material zu selten, um eine lückenlose Darstellung dieser Vorgänge geben zu können. Jedenfalls geht aus den vorliegenden Stadien bereits hervor, daf bei Caementella ähnlich wie bei anderen Tripyleen mehrere Arten der Teilung vorkommen können."

\section{Protocystis tridens und xiphodon Haeckel.}

Aus den Funden von gemeinsamen Vorkommen von Caementella und Challengeriden kann man schließen, daß für die Beziehungen dieser beiden Familien untereinander nur Protocystis tridens und Protocystis xiphodon in Frage kommen könnten. Aus den Funden, die Caementella in enger Beziehung mit einer Challengeride zeigen, ist stets ohne weiteres zu erkennen, daß es sich um eine Art handeln muß, die eine zarte Schale mit der üblichen Diatomeenstruktur hat, und außer dem Halsfortsatz keinerlei Fortsätze an der Schale besitzt. Von den beiden Protocystis-Arten lagen aber nur von Protocystis tridens die vollständigen Untersuchungen vor. Aus einigen von MrELcK gemachten Hinweisen ist aber zu schließen, daß die Beobachtungen an Protocystis xiphodon ebenfalls beendet waren. Leider war dieser Teil des Manuskriptes unter den nachgelassenen Aufzeichnungen 
nicht zu finden. Eine erneute Untersuchung dieser Art war nicht möglich, da nur einige wenige Präparate, die außerdem noch schlecht erhalten waren, zu finden waren. Es kann deshalb nur dort, wo Protocystis xiphodon erheblich abweicht im Bau von Protocystis tridens, auf diese Unterschiede hingewiesen werden. Im übrigen sind sich diese Arten auch so ähnlich, daß man bei einem Protocystis Exemplar, welches keinen Halsfortsatz besitzt, nicht ohne weiteres sagen kann, ob es sich um Protocystis tridens oder xiphodon handelt. Protocystis tridens ist jedoch im Durchschitt nach den Angaben verschiedener Autoren etwas kleiner (Schalendurchmesser 0,06-0;1 mm) als Protocystis xiphodon (Schalendurchmesser $0,09-0,13 \mathrm{~mm}$ ). Auch sind im allgemeinen die Maschen der Diatomeenstruktur bei xiphodon etwas gröber als bei tridens.

\section{Der Bau von Protocystis tridens.}

a) Die Zentralkapsel.

„Die Zentralkapsel besitzt im Unterschied zu Protocystis xiphodon im Verhältnis zur Größe des Gehäuses einen nur geringen Umfang und eine meist wenig scharf umschriebene, unregelmäßige Gestalt, vergl. Abb. 6 a u. b, 8 mit 7 . Sie hat ihren Platz gewöhnlich am Grunde der Schale der dorsalen Wandfläche fest angepreßt, vergl. Abb. 6a u. b.
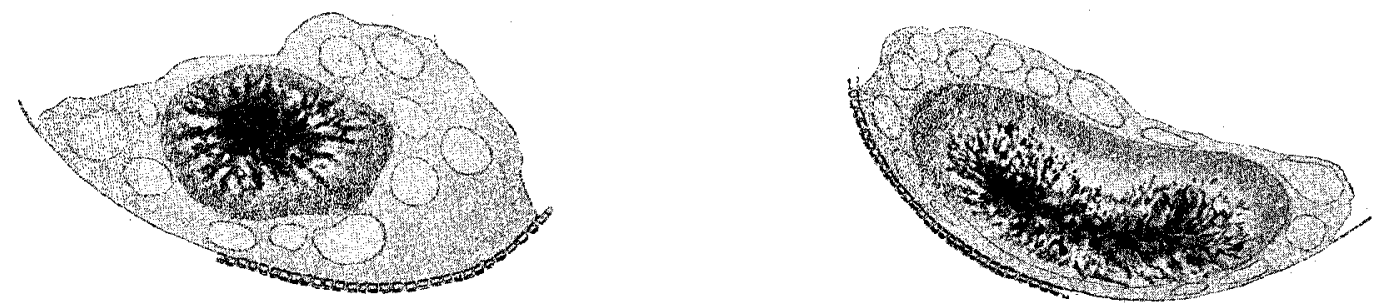

Abb. 6 a u. b. Protocystis tridens: Verschiedene, häufig auftretende Kernstrukturen. Haem. EHRLrcH, Eosin. Vergr. $840 X$.

Oft ist sie entweder in der Längs- oder in der Querachse des Gehäuses etwas in die Länge gezogen. Oefters liegt sie frei in dem Hohlraum und zeigt dann mehr kugelige oder ellipsoide Gestalt. Die Beschaffenheit der Kapselmembran ließ sich nur schwer genau untersuchen, es gelang nie eine doppelte Natur derselben festzustellen; ihr Vorhandensein wurde überhaupt in den seltensten Fällen deutlich, am leichtesten, wenn die Zentralkapsel frei im Gehäuse lag. Hatte sie aber ihren Platz dorsal an der Wandfläche der Schale, so erkannte man sie nur an der Stelle, wo die Zentralkapsel anlag, während an den dem Phaeodium zugekehrten Stellen das Protoplasma der Zentralkapsel sich zwischen den Phaeodellen zu verlieren schien. Das Vorhandensein einer Astropyle konnte ich ebenfalls nicht mit Sicherheit feststellen, noch viel weniger kann ich über das Vorhandensein von Parapylen etwas aussagen ${ }^{1}$ ).

Sehr auffällig ist die Tatsache, der ähnliche Verhältnisse auch bei Protocystis xiphodon entsprechen, daß ungefähr die Hälfte aller untersuchten Protocystis-Gehäuse äberhaupt keine Zentralkapseln, sondern meist nur in großen Mengen Phaeodellen enthielt (Abb. 9). Dabei handelt es sich sonderbarerweise nicht nur um völlig ausgebildete, vielleicht verlassene Gehäuse, sondern um Schalen, die nach ihrer ganzen Struktur und Färbbarkeit ganz den Eindruck von Neubildungen machen. Bei letzteren handelt es sich vielleicht um Exemplare, die, eben gebildet, sich zufällig von dem Zusammenhang losgelöst haben, ehe sie bezogen waren. Es wird in dem nächsten Abschnitt noch auf diese Erscheinung zurückzukommen $\left.\operatorname{sein}^{2}\right)$.“

1) Verfasserin konnte eine Astropyle sowohl bei Protocystis tridens als auch bei Protocystis xiphodon einmal nachweisen. Es handelt sich dabei um Gebilde von einfachstem Bau, vergl. Abb. 7. Es ist deshalb wohl anzunehmen, daß dieselben stets vorhanden sind, aber fast stets durch die zahlreichen, die Zentralkapsel dicht umgebenden Phaeodellen verdeckt werden.

2) Nach den Beobachtungen, die die Verfasserin an dem reichen Protocystis tridens-Material machen konnte, das der Fang vom März 1935 enthielt, ist die ZahI der Gehäuse, welche wohl zahlreiche Phaeodellen enthalten, aber einer Zentralkapsel entbehren, nicht immer so häufig, wie MIELCK angiebt. Derartige Exemplare waren hier sogar ziemlich selten. 
b) Das Endoplasma.

„Das Endoplasma besitzt die für die Tripyleen typische alveoläre Struktur, meist sind die Alveolen sehr groß, so daß ein schaumiges Aussehen entsteht (s. Abb. 6 a und b; 8), seltener zeigt sich ein ganz feiner alveolärer Bau. Das Endoplasma erinnert sehr an Caementella, indem das Plasma selbst nur in Gestalt eines Netzwerkes zwischen den Alveolen vorhanden ist, das gleiche gilt für Protocystis xiphodon. Bei zwei Exemplaren konnte ich im Endoplasma Bildungen wie die bei Phaeocolla pygmaea beschriebenen "Chromidienbläschen" feststellen. Sie fanden sich in ganz geringer Zahl und waren in einer Vakuole des Endoplasma eingeschlossen. Ihre Größe betrug nur etwa 0,003--0,004 mm, also auch nicht größer als bei Phaeocolla. Das Bläschen selbst enthielt zahlreiche sehr kleine Chromatingranula. In beiden Fällen war auch das Chromatin des Kernes in zahlreiche Brocken aufgelöst und zeigte die sogenannte „Schollenstruktur", s. Anm. ${ }^{\text {). }}$.

Die intrakapsulären Teile des Weichkörpers scheinen sehr vergänglich zu sein, denn nur in seltenen Fällen sind Andeutungen davon zu finden. Als solche kann man vielleicht einen zart färbbaren Belag an der inneren Gehäusewand ansehen. Zuweilen konnte man auch zwischen den Phaeodellen in der Nähe der Zentralkapsel Weichkörperteile vermuten, indem einzelne Phaeodellen in eine abstehende Vakuole eingeschlossen waren, die von färbbaren Weichkörperteilen umschlossen war."

\section{c) Der Kern.}

„Der Kern zeigt kugelige bis ellipsoide Gestalt und besitzt meist eine deutliche Membran. Befindet sich die Zentralkapsel in der gewöhnlichen Lage an der dorsalen Wand des Gehäuses, so ist der Kern in derselben Weise wie diese in dorsiventraler Richtung abgeplattet, s. Abb. 6 b. Das Chromatin liegt im zentralen Teil des Bläschens, während die peripheren von nicht färbbaren Bestandteilen des Kernes eingenommen werden; s. Anm. $\left.{ }^{2}\right)$.

Am häufigsten, besonders wenn sich die Zentralkapsel in Ruhelage befindet, zeigte das Chromatin eine radiäre Anordnung mit meist freiem, seltener dichtem Zentrum und besaß eine homogene Konsistenz. Sie erinnert an HAECKERS "Radstruktur". Nicht selten löst sich diese Struktur in kugelige oder längliche Brocken auf, die bald mehr radiär angeordnet waren, bald ungeordnet im ganzen Kernraum durcheinanderlagen, diese Erscheinung entspricht wohl HAECKERS Schollen- oder Strangstrukturen, vergl. die Abb. 6 a u. b; 8 .

Der Kernbau von Protocystis xiphodon ist hiervon verschieden; das leichte, oft beinahe elegante und zierliche Netzwerk, das für den Kern von tridens so typisch ist, findet sich hier niemals. Wohl ist das Chromatin auch hier radiär gerichtet, aber in dicken, oft schwer erkennbaren Strängen, wie das bei Caementella oft anzutreffen ist. Der Kern wirkt deshalb im Ganzen kompakter als bei tridens. Er ist außerdem, ebenso wie die Zentralkapsel, oft rund und liegt mit der letztgenannten meist in der Mitte der Schale. Man findet

ihn nie einseitig der Schalenwand fest angedrückt, wie bei Protocystis tridens. Ein anderes Stadium, auf dem eine Astropyle gut zu erkennen war, zeigt den Kern langgestreckt, wie er bei Caementella häufiger gefunden wurde, Abb. 7. Daß die Kernstruktur bei Proto-

1) Während die Verfasserin die Befunde nicht nachprüfen konnte, da weder eine Skizze noch ein Präparat zu finden war, die sich auf diese Beobachtungen bezogen, konnten die "Chromidienbläschen" bei Caementella gefunden und für Phaeocolla nachgewiesen werden, dab diese Gebilde aus dem Kern stammen (s. S. 145. ff.)

2) Verfasserin hält diese Anordnıng des Chromatins nicht für natürlich, sondern für ein Fixierungsprodukt. 
cystis tridens eine andere ist als bei Caementella, hatte schon SchröDER erkannt, obwohl er annahm, daß diese beiden Formen miteinander in engerer Beziehung stehen."

\section{d) Phaeodium.}

„Das Ph a e odium ist bald von größerem, bald geringerem Umfang. Es pflegt einer Seite der Zentralkapsel dicht anzuliegen. Die Phaeodellen sind durchweg bedeutend größer als bei Phaeocolla pygmaea, bei Caementella und bei xiphodon.

Die Phaeodellen sind wie von einer feinsten Membran, die gefaltet und zusammengefallen zu sein pflegt, umschlossen, bald mit tiefschwarzen Körnchen von verschiedenster Anzahl erfüllt, oft auch ganz leer, vergl. Abb. 8, 9. Sehr selten sieht man in diesen Phaeodellen andere Inhaltskörper, wie kleine Stückchen von Diatomeen oder andere unbestimm-

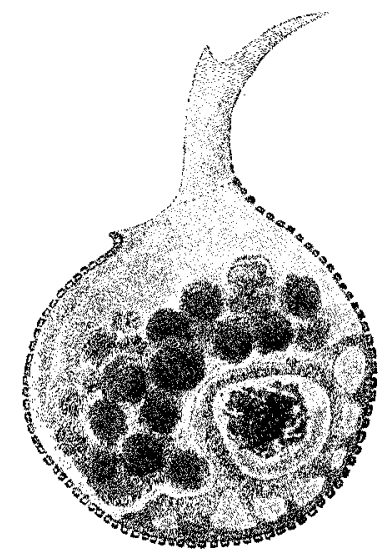

Abb. 8. Protocystis tridens: Die Zentralkapsel liegt dorsal der Schalenwand dicht an. Haem. Wergert, Eosin. Vergr. $420 \times$.

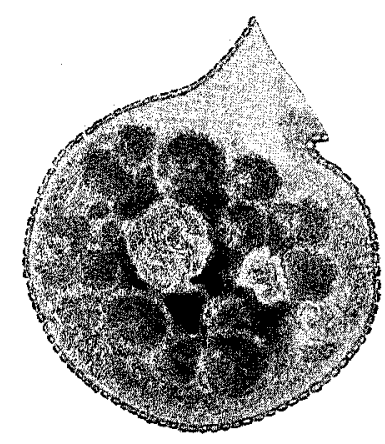

Abb. 9. Protocystis ohne Halsfortsatz, mit Phaeodellen und gefalteten Membranen angefüllt. Haem.WetGERT, Eosin. Vergr. $420 \times$.

bare kleine Bröckchen. Gelegentlich kamen im Phaeodium Dauersporen von Chaetoceras zur Beobachtung, doch lagen dieselben immer frei zwischen den Phaeodellen, nicht in diese eingeschlossen. Die Farbe der letzteren ist meist bräunlich, manchmal entbehren sie ganz der Farbe, abgesehen von den schwarzen Inhaltskörperchen. Farbstoff nehmen diese echten Phaeodellen im allgemeinen nicht an, im Unterschiede zu den übrigen Inhaltskörperchen der Schale, den Ballen und großen gefalteten Membranen unbestimmter Herkunft, die zum Teil reichlich Eosin annehmen. In einigen Fällen waren die Membranen als weiche strukturlose Tintinnengehäuse $\mathrm{zu}$ erkennen, manchmal waren es auch Häute von Copepoden. Diese Fremdkörper sind oft sehr groß, so daß schwer verständlich ist, wie dieselben durch die schmale Oeffnung den Weg in das Innere der Schale haben finden können."

\section{e) Die Schale.}

„Die Schale ist meist eben so lang wie breit. Es kommt jedoch häufig vor, daß diese entweder etwas länger als breit oder aber breiter als lang ist, wodurch die Gesamtform wesentlich geändert wird, obwohl die Differenz nur gering ist. Von den meisten Autoren (HAECKEL, HAECKER, BorgerT, SchröDER) wird die Schale als kreisrund beschrieben und nur der Durchmesser angegeben. Die Größe der Maße bewegt sich dabei zwischen $0,07-0,14 \mathrm{~mm}$. Der Halsfortsatz ist in einer Naht der schräg abgeschnittenen Mündung aufgesetzt. Diese Naht ist nicht immer deutlich, jedoch sieht man stets an der dorsalen Partie, wo die Schale am dicksten ist, und wo sich Fortsatz und Schale berühren, eine deutliche Einkerbung, vergl. Abb. 8. Daß die Schale an der Stelle, wo der Fortsatz dem Peristom ansitzt, sehr empfindlich ist, und wir es also tatsächlich mit einem sekundären Gebilde zu tun haben, zeigt die Erscheinung, da6 sich sehr oft Protocystis-Gehäuse finden, denen dieser Fortsatz fehlt. Es läßt sich dabei kaum nachweisen, $o b$ es sich um Exemplare handelt, die ïberhaupt keinen Hals ausgebildet haben oder um solche, denen er nachträglich durch irgendeine Verletzung abgegangen ist, s. Abb. 9. Eine große Anzahl der Schalen waren noch nicht vollständig ausgebildet; 
selten dagegen fanden sich Schalen, die noch ganz jung und völlig strukturlos waren und nur wie ein bei Druck nachgiebiges Bläschen in Form einer Protocystis waren. Bald fehlte solchen Stadien der Fortsatz ganz, bald war er als ganz zarte, durchsichtige, im dorsalen Teil doppelt konturierte Anlage zu erkennen. Solche weichhäutigen Schalen ließen aber immer bereits die doppelte Konturierung der Schale erkennen. Dabei hatte die innere Lamelle Farbstoff angenommen, die äußere nicht, wie mir das auch bei älteren Stadien aufgefallen ist. Nur in einem Falle, wo das Gehäuse gerade in Bildung war, zeigte die aborale Partie noch nicht die doppelte Kontur, während im dorsalen Teil der oralen Seite an einigen Stellen Andeutungen von Struktur zu sehen waren, die mit Bildern übereinstimmen, die HAECKER von Challengeria Naresi gibt (1904, II. Teil, p. 621, Abb. 195). Solche zarten Anlagen konnten auch gelegentlich bei ganz jungen Gehäusen beobachtet werden, die bei schwächerer Vergrößerung noch gänzlich strukturlos zu sein schienen. Bei diesen Formen sieht man auch bisweilen im dorsalen Teil der Schale, wo die Schalenbildung am dicksten zu sein pflegt, e ne feine Streifung. Etwas weiter ausgebildete Schalen, bei denen die Struktur bereits deutlich ist, sind oft noch sehr weich, so daßs bei Druck die Schale nicht zerbricht, und es bei geeigneten Manipulationen gelingt, die Schale zu zerreißen. Es gibt dann alle Uebergänge von weichen, halbspröden bis zu ganz starren, brüchigen Schalen. Auch solche, die bereits ausgeprägte Struktur haben, lassen sich noch bisweilen mit Haematoxylin, Paracarmin oder Eosin färben. Vollkommen ausgebildete, ungefärbte Schalen waren ziemlich selten.

Der Fortsatz ist also eine sekundäre Bildung, denn zuweilen fehlt den Schalen wie schon erwähnt wurde - derselbe vollständig, oder er zeigte eine noch weichere Konsistenz als die Schale, so daß er bei Druck in dem umgebenden Medium hin und her flutete. Dabei zeigte sich der Flügel und die Seitenzähne ganz besonders zart, sodaß man sie leicht übersehen kann, während der dorsale Teil kräftiger ausgebildet ist. Ein Fund deutet darauf hin, daß die Zentralkapsel durch Hervortreten an der Mündung nach Bildung der Schale diejenige des Fortsatzes übernimmt; der Fortsatz war noch ganz weich, besaf weder Struktur noch eine feste Verbindung mit der Schale. Dagegen war durch feine Gallertmassen ein Zusammenhang mit der Zentralkapsel vorhanden. Die Weichheit der Schale spricht dafür, daf es sich nicht um ein Auswandern der Zentralkapsel handeln kann, wie es BorGERT einmal in ganz ähnlicher Weise beschreibt (1909, p. 218). Für Protocystis xiphodon ist es nachweisbar, daß wir es mit einer Neubildung zu tun haben. Die Exemplare von dieser Art, die noch im Zusammenhang mit Caementella waren, und über die im nächsten Abschnitt ausführlich berichtet werden soll, sind zum großen Teil ohne Fortsatz, teilweise auch, wenn die Schale schon deutliche Struktur aufweist. Die Schalen scheinen sich von der Caementella erst dann zu lösen, wenn auch der Hals fertig ausgebildet ist, denn es wurden nie Exemplare gefunden, bei denen dieser Fortsatz noch nicht fertig oder zum mindesten weichhäutig war, wie das bei Protocystis tridens oft der Fall war. Alle gefundenen Exemplare von xiphodon waren in ihrer endgültigen Ausbildung".

\section{f) Fortpflanzungserscheinungen.}

Ueber Fortpflanzungsstadien fehlen Aufzeichnungen sowohl für Protocystis tridens als auch für Protocystis xiphodon. Unter den vorhandenen Präparaten fand sich nur ein Protocystis tridens mit zwei Zentralkapseln. Es sollen an dieser Stelle noch einige von MIELCK gemachte Notizen zusammengestellt werden, die auf eine enge Beziehung von Protocystis tridens mit einem Phaeocolla-Stadium schließen lassen. - „Eine auffällige Erscheinung ist es, daß fast der Hälfte aller beobachteten Protocystis tridens-Gehäuse die Zentralkapsel fehlte und zwar auch solchen (vergl. S. 150, Anm. 2), die durch ihre zarte oder fast fehlende Struktur und die Färbbarkeit sich als deutliche Jugendstadien erwiesen. Ein Phaeodium besaßen dieselben aber fast immer. Diese Befunde erinnern im hohen Grade an diejenigen von Protocystis xiphodon, wo, wie noch gezeigt werden soll, nicht selten an der Caementella Gehäuse gebildet werden oder wenigstens an derselben befestigt sind, die keine Zentralkapsel enthalten. Man könnte aber denken, daß es sich hier um Bildungen handelt, die auf mechanische Art bei der Behandlung des Fanges von dem sie bildenden Phaeocollastadium losgelöst sind. Dagegen spricht allerdings ein Fund, aus welchem man schließen kann, dab hier die Zentralkapsel sich direkt mit der Schale umgibt, während dagegen bei Protocystis xiphodon (s. u.) die Schale außerhalb der Caementella gebildet wird. Die Zentralkapsel fliebt dann in das neue Gehäuse. Von einer Abstreifung des Gehäuses kann deshalb nicht die Rede sein. Eine Erklärung dieser jugendlichen Gehäuse ohne Zentralkapsel und mit Phaeodellen läßt sich also vorderhand weder für Protocystis tridens noch für Protocystis xiphodon geben. 
Was weiter als auffallende Tatsache zu verzeichnen ist, ist die Erscheinung, daß von Protocystis tridens die skelettlosen Stadien so gut wie vermißt wurden, während sie von Protocystis xiphodon in großer Zahl vorlagen. Nur ein einziger Fund scheint uns das dazugehörige skelettlose Stadium vorzuführen in einer Phaeocolla von einfachstem Bau". - Obwohl Skizzen und auch Präparat zu diesem Fund fehlten, soll er der Wichtigkeit halber nicht unerwähnt bleiben. - Die Phaeocolla besteht aus einer mit Protocystis tridens im Bau der Zentralkapsel vollkommen übereinstimmenden kleinen Zentralkapsel, die von sehr großen Phaeodellen, wie wir sie von Protocystis tridens kennen, umgeben ist und außerdem in ihrem Kalymma mehrere große, mit Eosin gefärbte, gefaltete Membranen besaß, die sich bei näherer Untersuchung als zu Gehäusen von Tintinnus norvegicus gehörig erwiesen. Die Größenverhältnisse von Zentralkapsel, Kern und Phaeodellen und die Struktur des Kernes berechtigen zu der Annahme, daf ein Phaeocolla-Stadium vorliegt, das noch nicht begonnen hat ein Gehäuse zu bilden. Für das seltene Auftreten derartiger Stadien kann man dann wohl die Zartheit des Kalymma mit verantwortlich machen, das womöglich schon bei dem Druck während des Netzzuges auseinanderfällt. Caementella loricata bietet dem gegenüber ein viel günstigeres Fangobjekt, wovon die beschalte Form, also wahrscheinlich Protocystis xiphodon (s. u.), wiederum sehr selten angetroffen wurde. Aehnliches teilt auch Borgert für die von ihm in Neapel gemachten Fänge von Caementella mit (zul. 1922, p. 177).

Was die Bildungsweise der Schalen betrifft, so wird sie wahrscheinlich bei beiden Arten im Grunde dieselbe sein, daf also die häutige, blasenförmige Grundlage peripher um die Zentralkapsel abgeschieden wird, und zwar hätte man den einfachsten Fall bei Protocystis tridens, wo sich, worauf ein Fund hindeutet, die Schale um die ganze Phaeocolla mit der Zentralkapsel ungefähr als Mittelpunkt bildet, während bei Protocystis xiphodon die Verhältnisse durch Anwesenheit eines Fremdkörperskelettes etwas komplizierter lägen (s. u.).

\section{Die Beziehungen zwischen Caementella und Protocystis.}

Die in dem folgenden Abschnitt mitgeteilten Beobachtungen machen die bereits oben ausgesprochenen Vermutung im hohen Grade wahrscheinlich, daß die hier in Frage kommende Caementella loricata als besondere Form ohne Eigenskelett in den Entwicklungskreis einer anderen skelettragenden hineingehört, nämlich von Protocystis (xiphodon). Eine Anzahl von Exemplaren der Caementella loricata besaßen an ihrer Hülle die Schale einer Protocystis, und zwar steckte dieselbe stets mit ihrer Oeffnung in dem Gehäuse der Caementella und ließ keinen Fortsatz erkennen. Dabei waren diese Gehäuse sowohl ganz weichhäutig, leicht färbbar, doppelt konturiert und mit kaum erkennbarer Struktur als auch schwach verkieselt, zwar oft noch färbbar aber bereits strukturiert, und schließlich kaum oder überhaupt nicht färbbar und normal strukturiert. Diese Befunde würden zwar an sich nur einen kleinen Beitrag zum Beweise der Richtigkeit der ausgesprochenen Vermutung liefern, denn es könnten möglicherweise leere Protocystis-Schalen ebenso in das Gehäuse aufgenommen werden wie andere Radiolarien-Skelette auch. Doch es gelangten Zustände zur Beobachtung, die eine derartige Vermutung wohl rechtfertigen:

Bei einigen Exemplaren begannen die Zentralkapseln in die Schale einzuwandern, bei anderen vollzog sich gerade der Eintritt, und schlieflich wurden Protocystis-Gehäuse mit Tieren angetroffen, die an der Schalenöffnung noch Reste der Caementella-Hülle besaßen. Vielleicht ließe sich auch der Standpunkt vertreten, daß der Vorgang, den die beobachteten Stadien wahrscheinlich machen, ein umgekehrter sei, daß nämlich die Protocystis-Schale an der Caementella-Hülle als von dem jetzt in der Caementella-Hülle befindlichen Tiere verlassene Schale anzusehen sei. Doch sprechen die Befunde gegen eine solche Deutung. Zwar zeigen die Schalen der frei gefundenen Protocystis-Individuen in den Fängen, die reichlich Caementellen enthielten, allgemein eine verhältnismäßig schwache Verkieselung im Vergleich zu denen mancher anderer Fänge; doch die besondere Zartheit der meisten an den Caementellenhüllen befindlichen Schalen kennzeichnete dieselben deutlich als Neubildungen. Gleichfalls spricht dafür wohl das Fehlen eines Fortsatzes, der bei den an Caementellenhüllen haftenden Protocystis-Schalen niemals gesehen wurde und wohl deshalb schon auch als sekundäre Bildung angesehen werden muß. $\mathrm{Zu}$ Gunsten der zweiten Erklärung ließen sich allerdings auch einige Befunde geltend machen, doch weisen diese wohl eher in eine andere Richtung. An manchen Caementellen mit einer Zentralkapsel wurden zwei Protocystis-Schalen gleichzeitig gefunden. Bei den vorliegenden Stücken war trotz der Zartheit der Schalen die Entscheidung schwer, ob man es mit alten oder neuen zu tun hatte. Die Zentralkapsel war in diesen Fällen entweder im Begriff, in 
eine dieser Protocystis-Schalen überzutreten, oder sie befand sich schon in derselben. In der anderen Schale fanden sich dagegen größere Mengen von Phaeodellen angehäuft. Möglicherweise ließ sich hier an Kopulation denken. In der Tat zeigen die hier in Frage kommenden Zentralkapseln alle auffällige Größe. Gegen diese Erklärung spricht jedoch das Fehlen des Fortsatzes an den Gehäusen. Vielleicht kommen beide Möglichkeiten in Betracht, indem nämlich sowohl Caementelliden mit Protocystis-Schalen vorkamen, wo die Schalen als Neubildungen, wie auch solche, wo sie als verlassene Schalen anzusehen sind. Denn es ist immerhin möglich, daß der Fortsatz sich zwischen den Hüllteilen der Caementella zuweilen der Beobachtung entziehen konnte.

Wie bereits angedeutet, muß die Frage nach der eigentlichen Bedeutung des Verhältnisses von Caementella zu Protocystis noch offen bleiben. Möglicherweise gibt es mehrere Vorgänge, die zur Bildung eines Caementella-Stadiums führen. Es dürfte sich daher wohl empfehlen, die verschiedenen Stadien dieser Befunde einzeln zu beschreiben:

a) Die Zentralkapsel von Caementella wandert in eine Protocystis-Schale ein.

1. Die Caementella besitzt an ihrer Hülle eine Protocystis-Schale mit zarter, oft kaum erkennbarer Struktur, doppelt konturiert, die so weich ist, daß in ihr Faltenbildung beobachtet wurde und dieselbe bei Druck mit dem Deckglas eher zerrif als zerbrach. In den meisten Fällen befanden sich in der Schale Phaeodellen, und zwar entweder einige wenige oder die Hauptmasse des Phaeodiums der Caementella (vergl. Abb. 10). Einmal ragte der größte Teil des Phaeodiums, von einer Membran umschlossen, eine kugelige Masse bildend, in die Protocystis-Schale hinein. Scheinbar war dieses Phaeodium durch die es umgebende Membran mit der Zentralkapsel verbunden. Ein kleiner Teil lag lose außerhalb der neuen Schale; die Zentralkapsel, welche sich in diesem Stadium in der Caementellahülle befand, hatte eine solche Lage, daß ihre Längsachse auf den Eingang der Protocystis-Schale gerichtet war (wie bei Abb. 13 links zu sehen ist). Einige Exemplare zeigten eine fortsatzartige Verlängerung eines spitzen Endes der Zentralkapsel, die sich nahe an der Protocystis-Schale befanden. Diese Stadien leiten hinüber zu der nächsten Gruppe, bei der ein Teil der Zentralkapsel sich außerhalb der Schalenmündung, der andere sich innerhalb derselben befindet.

2. Von der Zentralkapsel befindet sich ein Fortsatz innerhalb der Schale der Protocystis, in der einige Phaeodellen sich befinden, der größte Teil außerhalb derselben. Auf einem weiteren Stadium steckt die Hälfte der Zentralkapsel in der Schale, die andere noch in dem Caementella-

Gehäuse (vergl. auch Abb. 16). In der Mündung der Schale ist die Zentralkapsel eingeschnürt. Ein drittes Exemplar steckt fast ganz in der Protocystis-Schale, nur ein kleiner Teil der Zentralkapsel, die in der Schalenmündung eingeschnürt ist, befindet sich noch in der Caementella-Hülle, vergl. Abb. 11. Die beiden letzten Zustände besaßen in der Schale keine Phaeodellen.

3. In diesem Stadium befindet sich die Zentralkapsel ganz in der Protocystis-Schale. Die Schale eines dieser Exemplare erwies sich als blasige, strukturlose Haut, von der Form einer Protocystis. Zentralkapsel und Phaeodium befanden sich in derselben. Hier muß die Zentralkapsel schon früh in eine ganz junge Schale eingewandert sein. Bei einem anderen Tier war die Zentralkapsel vollständig in dem Protocystis-Gehäuse, an der Schalenmündung - der Fortsatz fehlte noch gänzlich - befanden sich die Reste des CaementellaGehäuses und Phaeodiums. In der Protocystis-Schale waren noch keine Phaeodellen (s. Abb. 12).

4. Vollständig ausgebildete Protocystis-Individuen, die einen Fortsatz, Zentralkapsel und Phaeodium haben, verraten durch den Besitz größerer oder kleinerer Massen der Caementella-Hüllbilden an ihrer Mündung ihren Zusammenhang mit solchen (vergl. Abb. 14). 
5. Eine Besonderheit stellt noch folgender Fall dar:

Ein Caementella-Gehäuse enthielt zwei Zentralkapseln, ihm liegt seitlich eine große, ganz zart strukturierte Protocystis-Schale an, vergl. Abb. 13. Es ist deutlich zu erkennen, daß die eine Zentralkapsel sich anschickt in die Protocystis-Schale einzuwandern, in der sich bereits einige Phaeodellen befinden. Dabei liegt im Unterschied zur gewöhnlichen Zwei-

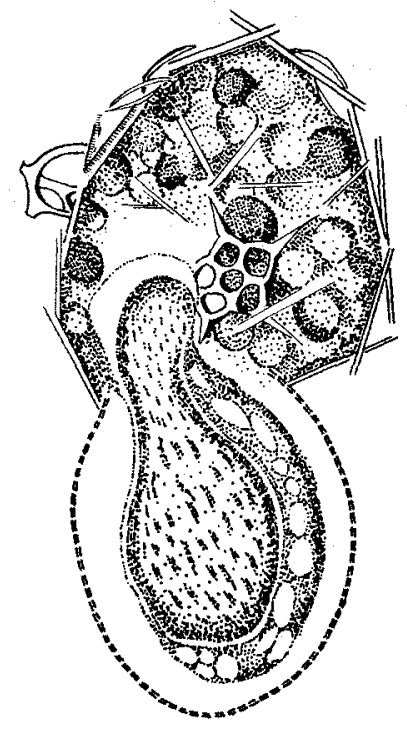

Abb. 11. Caementella-Gehäuse mit zahlreichen Phaeodellen. Protocystis - Schale mit Zentralkapsel, die nur noch mit einem kJeinen Teil in dem Caementella-Gehäuse steckt. Nach einer Skizze von Prof. MiELCK. Haem. EHrLICH, Eosin.

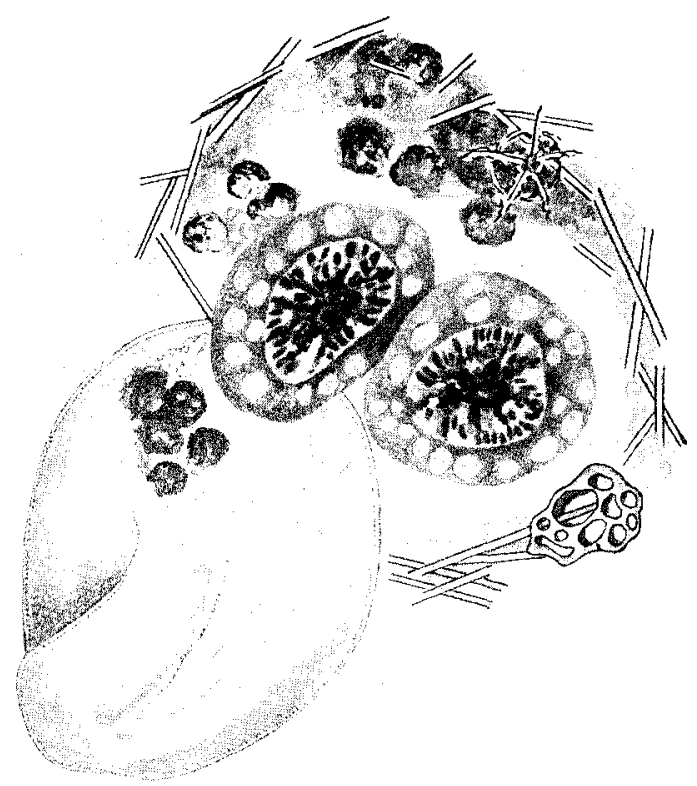

Abb. 13. Caementella-Gehäuse mit zwei Zentralkapsel, von denen eine sich anschickt in eine ganz junge, gefaltete ProtocystisSchale einzuwandern. Haem. EHRLtch. Vergr. $420 \times$.

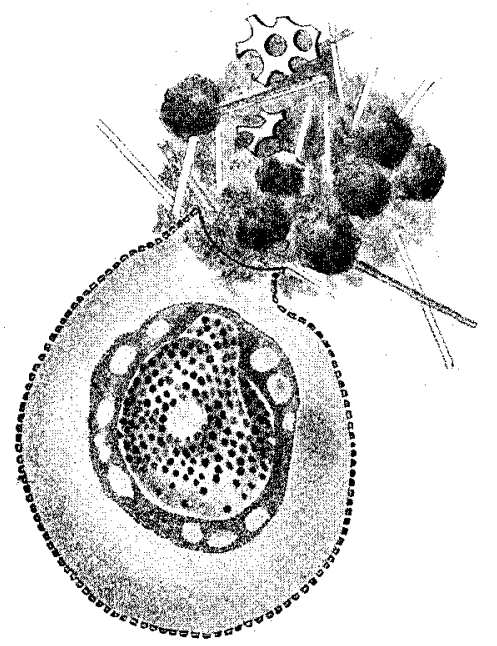

Abb. 12. Zarte Protocystis-Schale mit Zentralkapsel. Der Kern hat noch einen Fortsatz, und das Chromatin ist in einzelne Granula aufgelöst. Im Kern ist ein eigenartiger, chromatinloser Hohlraum ausgebildet. Paracarmin. Vergr. $420 \times$.

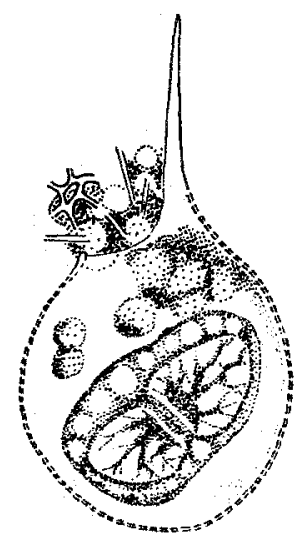

Abb, 14. Protocystis xiphodon mit Resten eines Caementella-Gehäuses am Hals. Die Zentralkapsel enthält zwei Kerne, die eben entstanden sein müssen. Die radiëre Struktur des Chromatins ist noch nicht wieder ausgebildet. Nach einer Skizze von Prof. MIELCK. Paracarmin. 
teilung bei Caementella das Phaeodium nicht zwischen den beiden Zentralkapseln - an denen übrigens keine Astropyle zu erkennen ist. Dort hat es ja den Anschein, als wenn es bei dem sich teilenden Fremdkörperskelett die offene Seite zunächst abschließt (Körbchenform MrELcks), bis die andere. Hälfte des Gehäuses von außen ergänzt wird. Hier jedoch liegt das Phaeodium der zarten Protocystis-Schale direkt gegenüber und ist von dieser durch die beiden Zentralkapseln getrennt. Dieselben befinden sich mit ihrer Schmalseite einerseits an der Protocystis-Schale, andererseits an dem Phaeodium. Da die Kerne der Zentralkapseln schon wieder die Struktur der ruhenden Caementella haben, ist auch nicht anzunehmen, daß das Phaeodium seine Lage noch verändert; dagegen spricht auch, daß bei der gewöhnlichen Zweiteilung von Caementella (vgl. Abb. 4) das Phaeodium bereits zwischen den Zentralkapseln liegt, wenn die Kerne der Tochterindividuen noch in dem Anaphasenstadium sind. Es gibt für diesen Befund mehrere Erklärungsmöglichkeiten. Einmal kann man annehmen, daß es neben der gewöhnlichen Zweiteilung von Caementella, bei der zwei neue Caementella-Individuen mit je einem halben Gehäuse entstehen, noch eine andere gibt, bei der neben dem vorhandenen Caementella-Gehäuse, das von dem einen Tochtertier bewohnt bleibt, eine Protocystis-Schale für das andere Tochtertier ausgebildet wird. Es ist aber auch möglich, daß bei den weiter unten zu schildernden Fällen noch eine Protocystis-Schale ausgebildet wird, dje dann von der anderen Zentralkapsel bezogen wird. Das Caementella Gehäuse wäre dann überflüssig. Der Wechsel des Gehäuses geht also auch hier von Caementella zu Protocystis.

\section{b) Ist Caementella stets die primär vorhandene Form?}

Während bisher stets Caementella das primär vorhandene war, und Protocystis als das Folge-Individuum entstand, liegen nun noch Exemplare vor, bei denen der Wechsel des Gehäuses in der eben erwähnten Reihenfolge nicht ohne weiteres sicher ist. Es handelt sich dabei bei dem einen um das einzige Exemplar, wo der Hals so weit deutlich zu erkennen ist, daß man mit Sicherheit die Art als Protocystis xiphodon erkennt. Die Struktur der Schale ist in endgültiger Ausprägung vorhanden. Die Zentralkapsel enthält zwei Kerne in dem unteren Schalenabschnitt. Am Hals der Protocystis-Schale sind Phaeodellen und ein Distephanus-Skelett in dichter Ansammlung wie bei dem Rest eines CaementellaGehäuses. Einfache Zweiteilung tritt nun zum Teil schon auf, wenn die Protocystis-Schale noch ganz zarte Struktur hat, die Zentralkapsel also eben erst eingewandert sein kann, und das Caementella-Gehäuse noch in aller Deutlichkeit vorhanden ist. Hier jedoch ist vorläufig auch noch die Möglichkeit ins Auge zu fassen, daß ein Vorgang in umgekehrter Reihenfolge vorliegt, daß also die Protocystis-Schale das Primäre ist, und an dem Hals derselben ein Caementella-Gehäuse für die neu entstehende Zentralkapsel gebildet wird. Eine derartige Annahme ist schon deshalb nicht von der Hand zu weisen, als noch nie ein Stadium gefunden wurde, wo eine Protocystis mit zwei Zentralkapseln eine neue Schale für das Tochterindividuum bildet. Bei völlig freien Zentralkapseln, die auch kein Phaeodium besitzen, ist ja nie festzustellen, ob es sich nicht um Tiere handelt, die nur gewaltsam ifi: Gehäuse verloren haben, vergl. hierzu Abb. 14.

Bei den folgenden Exemplaren, die ebenfalls Beweisstücke für die Beziehungen von Protocystis und Caementella sind, ist es weit schwieriger, etwas Sicheres über den Verlauf der Vorgänge auszusagen. Es handelt sich um drei verschiedene Stadien, die einzeln beschrieben werden sollen. Das Gemeinsame, was sie alle auszeichnet, ist die Tatsache, dali auBer einem mehr oder weniger vollständigen Caementella-Gehäuse stets zwei Protocystis-Schalen vorhanden sind.

\section{c) Caementella-Gehäuse mit zwei Protocystis-Schalen.}

1. Stadium I: Ein Caementella-Gehäuse mit verhältnismäBig großem Innenraum enthält eine große Zentralkapsel und wenig Phaeodellen, s. Abb. 15. Thm liegt an einer Seite eine Protocystis-Schale an, die fast vollständig mit Phaeodellen angefüllt ist. Die Struktur der Schale ist bei diesem Exemplar noch ganz zart. Dieser Protocystis-Schale gegenüber liegt, durch die Zentralkapsel der Caementella getrennt noch eine Schale, die bereits ausgeprägte Wandstruktur, aber nur wenig vereinzelte Phaeodellen enthält. Die Zentralkapsel hat in die letztgenannte Schale einen Plasmafortsatz hineingeschoben, und der Kern derselben schickt sich an überzufließen. Der Kern hat zwar schollige Struktur, aber die Schollen sind kurz zylindrisch und radiär gerichtet. Die Anordnung des Chromatins verrät vielleicht 


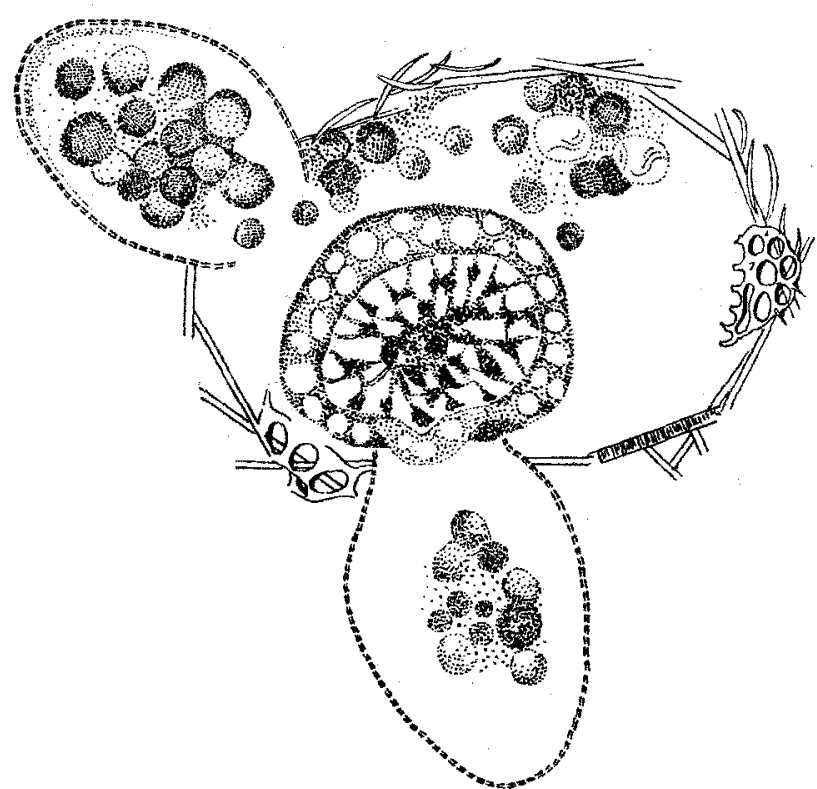

Abb. 15. Stadium I: Caementella mit zwei seitlich anliegenden Protocystis - Schalen, von denen eine nur Phaeodellen enthält, während in die andere die Zentralkapsel der Caementella einwandert. Nach einer Skizze von Prof. Miesck. vorausgegangene Mitose. Hierzu schreibt MrELcK noch, daß das geräumige Caementella-Gehäuse eventuell darauf schließen läßt, daß vorher zwei Zentralkapseln vorhanden gewesen sind, und, da das Gehäuse ja noch vollständig erhalten ist, vielleicht Kopulation vorausgegangen sein könnte. Da jedoch die Zentralkapsel auffallend groß ist, der Kern außerdem die bei dem Ruhestadium übliche Struktur besitzt, wäre es ja auch möglich, daß hier ein beginnendes Teilungsstadium vorliegt. Die Vorgänge der Kernteilung sind ja hier infolge des geringen Vergleichsmaterials noch so wenig in allen Einzelheiten bekannt, auch ist die Fixierung für derartige Untersuchungen nicht genügend, um definitives aussagen $z u$. können. Diese Fragen können wohl bei diesen kompakten Kernen nur durch Schnitte entschieden werden. Es wäre immerhin möglich, daß durch eine jetzt erfolgende Teilung von Kern und Zentralkapsel auch die andere Protocystis-Schale, die vorläufig nur mit Phaeodellen gefüllt ist, noch eine Zentralkapsel bekommt. Dann wäre aber schwer erklärlich, warum die Zentralkapsel mit Plasma und Kern bereits beginnt, in die eine Schale einzuwandern! Auf jeden Fall ist auch hier Caementella das zuerst vorhandene Individuum, da für die zart strukturierten Protocystis-Schalen feststeht, daß es sich um eine Jugendform handelt.

2. Stadium II : Die bei diesem Exemplar vorhandenen zwei Protocystis-Schalen haben vollständig ausgebildete Wandstruktur. Die eine Schale ist wie bei dem ersten Stadium mit Phaeodellen angefüllt, die andere jedoch enthält überhaupt keine Phaeodellen, dafür finden sich hier ungefähr zwei Drittel der Zentralkapsel und des Kernes, während das andere Drittel noch in dem Caementella-Gehäuse ist, welches durch die sich gegenüberliegenden Protocystis-Schalen stark eingeengt ist. Eine am Hals der Protocystis sichtbare Einschnürung verdeutlicht noch die ungleichen Abschnitte der Zentralkapsel. Der Kern von ansehnlicher Größe macht mit seinen Chromatinbrocken, die in nichts mehr an die eigentliche Kernstruktur erinnern, ganz den Eindruck des Ueberfließens, hierzu vergl. Abb. 16.

Nicht unerwähnt lassen möchte ich eine merkwürdige, scharf umgrenzte Oeffnung im Kern, von der nicht entschieden werden kann, ob es sich um eine Erscheinung handelt, die durch das Konservieren hervorgerufen worden ist, oder ob sie tatsächlich eine bei diesem Vorgang auftretende Besonderheit an dem Kern darstellt. Für die letztere Möglichkeit spricht auch, daß bei einem anderen Exemplar (s. Abb. 12) die gleiche Erscheinung $\mathrm{zu}$ beobachten ist. Auf eine große im Phaeodium der Caementella liegende gefaltete Membran soll auch noch hingewiesen werden (ist auf der Abb. infolge der Einstellung auf den Kern nicht zu sehen).

3. Stadium III : Zwei wohlausgebildete, d.h. mit ausgeprägter Struktur versehene Protocystis-Schalen sind durch ein Caementella-Gehäuse, das aus zahlreichen Distephanusringen und Phaeodellen besteht, zusammengehalten. Wie im vorhergehenden Exemplar enthält auch hier das eine Protocystis-Individuum die Zentralkapsel, das andere ist dagegen vollständig mit Phaeodellen ausgefüllt. Wenn sich auf dem vorhergehenden Stadium Kern und Zentralkapsel nicht mehr teilen, so bildet das hier vorliegende die direkte Fortsetzung zu dem eben besprochenen, s. Abb. 17.

In allen drei besprochenen Stadien ist also der Uebergang einer Zentralkapsel aus dem Caementella-Gehäuse in die Protocystis-Schale zu erkennen, ohne daß Zweifel daran geknüpft werden können. Es wiederholt sich somit der unter 1.-5, beschriebene Vorgang. Unerklärlich an diesen Exemplaren ist nur die zweite Protocystis-Schale, die wohl voller Phaeodellen ist, aber, wenn man von Stadium III. zu I. zurückverfolgt, keine Zentralkapsel vorkommt. Während man bei Stadium 1. und auch noch bei II. annehmen kann, daß die 
Zentralkapsel noch geteilt wird, ist das bei Stadium III. im höchsten Grade unwahrscheinlich, da die Zentralkapsel ja bereits restlos in die eine Protocystis-Schale übergesiedelt ist. Betrachten wir die Stadien I. und III. dagegen allein, so wäre die Möglichkeit einer Kopulation die bei I. dann schon vollzogen wäre - nicht von der Hand zu weisen.

Während die Stadien 1.-5. ohne weiteres dafür sprechen, daß die Zentralkapsel von Caementella zu Protocystis übersiedelt, ist das bei den zuletzt beschriebenen Exemplaren nicht von vornherein sicher.

Bezüglich der Bildungsweise der Schale liegen bei Protocystis xiphodon die Verhältnisse etwas schwieriger als bei Protocystis tridens durch die Anwesenheit eines Fremdkörperskeletts. Die Protocystis kann weder das umfangreiche Fremdkörperskelett mit in ihre Schale einschließen, noch ist es für sie günstig, im Innern desselben das Skelett auszubilden, denn dadurch, daß oft nicht genügend Platz sein dürfte, würde sie später Gefahr laufen, um ihr Gehäuse noch den alten Fremdkörpermantel der Caementella mit sich

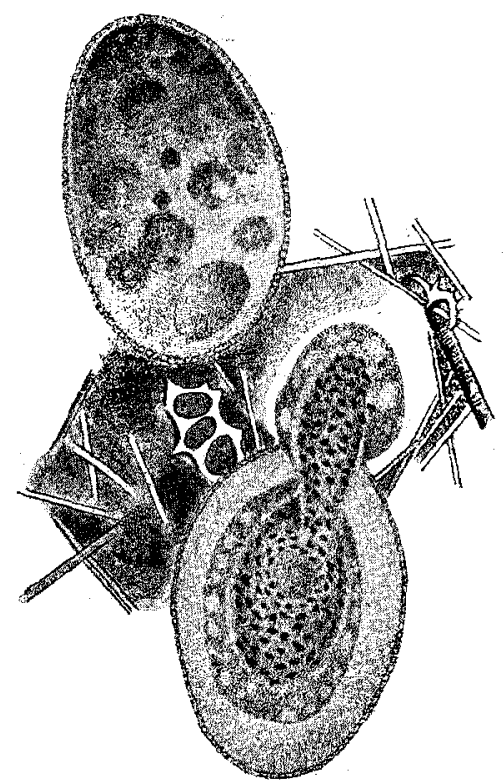

Abb 16. Stadium II: Caementella mit zwei seitlich anliegenden Protocystis-Schalen, von denen nur eine Phaeodellen enthält, während in die andere die Zentralkapsel überfließit. Der Kern zeigt ebenfalls den auf Abb. 12 sichtbaren Hohlraum, der frei von Chromatin ist. Paracarmin. Vergr. $420 \times$.

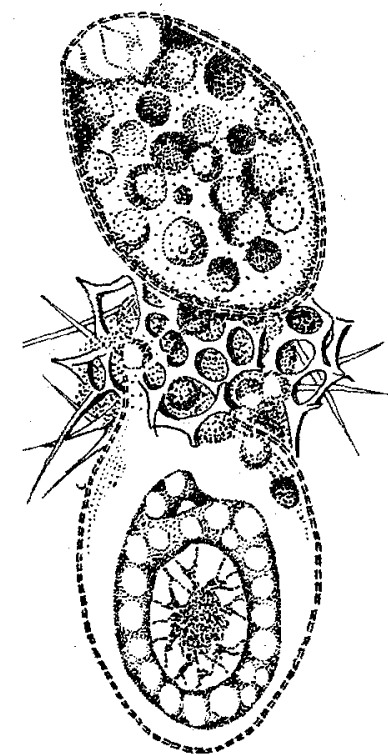

Abb. 17. Stadium III: Zwei Protocystis-Schaien, von denen die eine nur Phaeodellen und eine gefaltete Membran enthält, die andere die Zentralkapsel und wenig Phaeodellen. Beide Schalen werden durch die Reste eines Caementella-Gehäuses zusammengehalten. Nach einer Skizze von Prof. Mrelck Haem. EhrLich.

herumschleppen zu müssen. Es bleibt ihr also nichts anderes übrig, als die Gehäuseanlage aus der Schale herauszuschieben; doch wird man, glaube ich, auch hier annehmen müssen, daß die häutige Anlage die Zentralkapsel schon mit einschließt, nur daß letztere nicht im Zentrum derselben liegt, sondern ganz an dem einen Ende, und daß die Zentralkapsel nach Fertigstellung der Schale nachfließt. Man könnte noch denken, daß die Schale hier ganz außerhalb der. Zentralkapsel nur im Kalymma gebildet wird, ohne daß es die Zentralkapsel mit umschließ3t, doch wäre das Verhalten von dem bei Protocystis tridens erheblich abweichend und darum das Unwahrscheinlichere; außerdem wäre es wohl kaum einfacher. Doch sind bislang leider keine beweisenden Funde für die eine oder die andere Tatsache vorhanden.

\section{E. Zusammenfassung.}

Caementella loricata Borgert ist eine in der Nordsee nicht seltene Tripylee mit einem Fremdkörperskelett. Sie besitzt ein vakuolenreiches Endoplasma, in dem sich verstreut (in den Vakuolen) "Chromidienbläschen" finden. Für letztere konnte für Phaeocolla pygmaea nachgewiesen werden, daß sie aus dem Kern stammen. Das ausschlieflich extrakapsular gelegene Phaeodium besteht aus großen Phaeodellen, die der oralen Seite der Zentralkapsel anliegen. Der Kern zeigt die von HAECKER und BORGERT bereits ausführlich be- 
schriebene „Schollen“- oder „Radstruktur“. Eine Astropyle ist vorhanden, Parapylen fehlen jedoch. Fortpflanzung durch Zweiteilung konnte in verschiedenen Modifikationen festgestellt werden. Das Fremdkörperskelett besteht aus verschiedenen Bauelementen und ist in seiner Zusammensetzung abhängig von dem jeweiligen Artenreichtum der Umgebung. Die Zentralkapsel von Caementella gleicht der von Protocystis xiphodon so weitgehend, daß bei den nachweisbaren engen Beziehungen von Caementella zu Protocystis angenommen werden darf, daß es sich um Protocystis xiphodon handelt.

Die Untersuchungen an Protocystis konnten eingehend nur an tridens gemacht werden. Diese Art besitzt eine kleine Zentralkapsel, die der Schalenwand dorsal meist dicht anliegt. Der Kern hat eine zierliche Struktur, das Chromatin ist radiär angeordnet. Die Phaeodellen liegen nur extrakapsular. Die Schale hat einen nachträglich gebildeten Fortsatz. Es finden sich sowohl bei tridens als auch bei xiphodon zahlreiche Alveolen im Plasma, in denen teilweise auch "Chromidienbläschen" beobachtet wurden.

Bei Protocystis xiphodon ist die Zentralkapsel stets in der Mitte der Schale gelegen. Der Kern zeigt „Schollen“- oder „Radstruktur, wie bei Caementella. Die Phaeodellen sind kleiner als bei tridens und gleichen ebenfalls denen von Caementella. Die Schale hat die bei den meisten Challengeriden übliche Diatomeenstruktur, die etwas gröber ist als bei tridens. Der Fortsatz der Schale wird erst ausgebildet, wenn die Schale schon fertig ausgebildete Wandstruktur besitzt. Von Fortpflanzungsstadien ist bei beiden Arten bisher nur Zweiteilung bekannt.

Es gibt bei diesen Tripyleen zwei sich abwechselnde Generationen, von denen die eine Caementella- oder Phaeocolla-Charakter besitzt und die andere in beiden Fällen eine Challengeride ist. Caementella steht nach den bisherigen Ergebnissen mit Protocystis xiphodon in Generationswechsel, während für tridens als skelettlose Form Phaeocollaähnliche Stadien in Frage kommen dürften.

Die Schale von Protocystis wird an einem Caementella-Gehäuse gebildet. Gegen die Annahme, daß Caementella aus einem Teilungsstadium von Protocystis xiphodon hervorgeht, sprechen die Beobachtungen, daß bei Exemplaren, die Caementella in engem Zusammenhang mit einer Protocystis-Schale zeigen, die letztere meist jung und ohne Halsfortsatz ist. Dem Gehäusewechsel kann eine Zweiteilung vorausgehen, oder sie erfolgt sofort nach Bezug des neuen Gehäuses.

Es ist aus den vorhandenen Befunden die Möglichkeit nicht von der Hand zu weisen, daß neben diesen einfachen Beziehungen zwischen Protocystis und Caementella oder Phaeocolla noch andere bestehen. Die Tatsache, daß an einem Caementella-Gehäuse zwei Protocystis-Schalen gefunden wurden, von denen allem Anschein nach nur die eine Schale eine Zentralkapsel bekommt, ist nicht ohne weiteres erklärlich. Eine Zufälligkeit, auf die diese Erscheinung ja auch zurückgeführt werden könnte, wird wegen der Seltenheit dieser Funde, aus denen überhaupt auf eine Beziehung dieser Arten zueinander geschlossen werden kann, für ausgeschlossen gehalten werden müssen, besonders da es sich nicht um einen Einzelfall handelt. Außerdem gibt es (nach MiELCK) so zahlreiche junge Protocystis-Exemplare, die nur Phaeodellen und keine Zentralkapsel enthalten, daß diese Erscheinung irgendeinen, bisher noch nicht erkennbaren Grund haben muß.

Außer diesen beiden Fällen, wo Challengeriden mit einer einfachen Tripylee in Generationswechsel stehen, sind innerhalb dieser Familien bisher keine sonstigen Beziehungen festgestellt worden. Es ist aber aus den geschilderten Beobachtungen anzunehmen, daß sich noch weitere Beziehungen finden lassen werden. Bevor jedoch die Verhältnisse dieser Familien zueinander noch nicht weiter geklärt sind, besteht meines Erachtens kein Grund, an der systematischen Stellung dieser Familien etwas zu ändern, d. h. die Caementelliden und Phaeodiniden als selbständige Familien aufzulösen. 


\section{Schrifttum.}

Borgert, A.: Ueber die Dictyochiden insb. über Distephanus speculum; sowie II. Studien an Phaeodarien Z. wiss. Zool. Bd. 51, 1891.

- Zur Fortpflanzung der Tripyleen-Radiolarien. Zool. Anz. Bd. 19, 1896 a.

- Fortpflanzungsverhältnisse der Tripyleen-Radiolarien. Verh. Dtsch. Zool. Ges. 1896 b.

- Untersuchungen über die Fortpflanzung der Tripyleen-Radiolarien, spez. von Aulacantha scolymantha I. Teil. Zool. Jahrb. Abt. Anat. Bd. 14, 1901.

- Die Tripyleen-Radiolarien des Mittelmeeres. Mitt. Zool. Stat. Neapel, Bd. 14, 1901.

- Untersuchungen über die Fortpflanzung der Tripyleen-Radiolarien spez. von Aulacantha scolymantha II. Teil. Arch. Prot. Bd. 14, 1909 a.

- Die Tripyleen-Radiolarien der Plankton-Exped.: Phaeodinidae, Caementellidae und Cannorrhaphidae. Ergebn. Planktonexp. d. Humboldt-Stiftung, Bd. 3 L. h. 7, 1909 b.

- Die Tripyleen-Radiolarien etc.: Challengeridae, daselbst $1911 \mathrm{~b}$.

- Die Tripyleen-Radiolarien: II: Allgemeiner Teil. Bau und Fortpflanzung der Tripyleen, daselbst 1923.

GEMEINHARDT, K.: Organismenformen auf der Grenze zwischen Radiolarien und Flagellaten. Ber. Dtsch. Bot. Ges. Bd. 49, 1931.

HAECKEL, E.: Grundriß einer allgemeinen Naturgeschichte der Radiolarien. 1887.

- Challenger-Report. Zool. Vol. 18, 1. u. 2. Teil, 1887.

- Die Acantharien und Phaeodarien. III. u. IV. Teil der Monographie der Radiolarien. 1888.

HaEcker, V,: Tiefsee-Radiolarien. Allgemeiner Teil u. spez. Teil. Wiss. Ergebn. d. Dtsch. Tiefsee-Exp. Bd. 14, 1908.

Hertwig, R.: Der Organismus der Radiolarien. 1878.

Hovasse, R.: Radiolaires et Silicoflagellates. CR. Ac. Sci. Paris, T. 193, 1931.

JiRovec, O.: Protozoenstudien II. Nuclealreaktion bei einigen Protozoen. Arch. Prot. Bd. 59, 1927.

MEYER, K.: Die geographische Verbreitung der Tripyleen-Radiolarien des Südatlandischen Ozeans. Wiss. Ergebn. Meteor 1925/27, 1933.

MIELCK, W.: Untersuchungen an Nordseeprotisten. I. Ueber Phaeocolla pygmaea Borgert. Wiss. Meeresunters. N. F. Abt. Helgoland, Bd. 10, 1912.

- Heliozoa und Radiolaria. Bull. Trim. Cons. Perm. Int. Expl. Mer. III. me Partie, 1913.

Reichenow, E.: Ergebnisse mit Nuklealfärbung bei Protozoen. Arch. Prot. Bd. 61, 1928.

SchröpteR, O.: Die Tripyleen-Radiolarien der Dtsch. Südpol. - Exped. Die Dtsch. Südpolar-Exped. Bd. 14, Zool. VI, 1913. 